\title{
Melliferous flora and pollen characterization of honey samples of Apis mellifera L., 1758 in apiaries in the counties of Ubiratã and Nova Aurora, PR
}

\author{
ELIZABETE S. SEKINE ${ }^{1}$, VAGNER A.A. TOLEDO ${ }^{2}$, MARCELO G. CAXAMBU ${ }^{1}$, SUZANE CHMURA ${ }^{1}$, \\ ELIZA H. TAKASHIBA ${ }^{3}$, MARIA JOSIANE SEREIA ${ }^{1}$, LUÍS C. MARCHINI ${ }^{4}$ and AUGUSTA C.C.C. MORETI ${ }^{5}$ \\ ${ }^{1}$ Universidade Tecnológica Federal do Paraná/ UTFPR, BR 369, Km 0,5, 87301-006 Campo Mourão, PR, Brasil \\ ${ }^{2}$ Universidade Estadual de Maringá/ UEM, Departamento de Zootecnia, \\ Av. Colombo, 5790, 87020-900 Maringá, PR, Brasil \\ ${ }^{3}$ Universidade Federal de Santa Maria (UFSM), Departamento de Ciências Florestais, \\ Av. Roraima, 1000, 97105-900 Santa Maria, RS, Brasil \\ ${ }^{4}$ Escola Superior de Agricultura Luiz de Queiroz, Esalq/USP, Departamento de Entomologia e Acarologia, \\ Avenida Pádua Dias, 11, 13418-900 Piracicaba, SP, Brasil \\ ${ }^{5}$ Instituto de Zootecnia, Rua Heitor Penteado, 56, 13460-000 Nova Odessa, SP, Brasil
}

Manuscript received on April 26, 2011; accepted for publication on July 6, 2012

\begin{abstract}
The aim of this study was to carry out a survey of the flora with potential for beekeeping in the counties of Ubiratã and Nova Aurora-PR through the collection of plants and pollen analyses in honey samples collected monthly. 208 species of plants were recorded, distributed in 66 families. The families that showed the major richness of pollen types were: Asteraceae, Myrtaceae and Solanaceae. Approximately 80 pollen types were found in honey samples, most of them were characterized as heterofloral. Cultivated plants, such as Glycine max (soybean) and Eucalyptus spp., were representative in some months of the year. Exotic species, such as Ricinus communis and Melia azedarach, were also frequent. However, over than $50 \%$ of the pollen types belong to native species of the region, such as Schinus terebinthifolius, Baccharis spp. Alchornea triplinervia, Parapiptadenia rigida, Hexaclamys edulis, Zanthoxylum sp. and Serjania spp., indicating the importance of the native vegetation for the survival of the colonies.
\end{abstract}

Key words: beekeeping, bee plants, floral resources, floristic survey.

\section{INTRODUCTION}

Brazil is a country of great beekeeping potential due to its diverse flowerings and adequate climate conditions making possible the management of beehives during the entire year. Beekeeping is an interesting activity to the rural producer because it requires little initial investment (Nogueira-Couto and Couto 2006).

Correspondence to: Elizabete Satsuki Sekine

E-mail: essekine@gmail.com
Plants and their associated insects present interdependence in their evolutionary history and tropical regions have greater diversity of plants with flowers than temperate regions. Therefore, bees from tropics with a rich diversity of food resources demonstrate fast answer to the changes in the availability of the food items and lesser constancy than species of temperate regions (Crane 1990). The mutual benefits between bees and plants depend on the interaction with factors such 
as phenology and biogeography. In general, these factors are specific of each place and depend on the seasonal patterns (Hill and Webster 1995).

As the honey production and the parameters of honey depend on the vegetal species available to the beekeeping use, many studies have been carried out for the identification of the plants used by A. mellifera in different regions of the country. The authors employ mainly techniques based on the analysis of the pollen content of honey samples and/or analysis of pollen loads from corbicula of bees (Carvalho and Marchini 1999, Moreti et al. 2000, Silva and Absy 2000, Bastos et al. 2003, Viana et al. 2006, Luz et al. 2007, Mendonça et al. 2008, Sodré et al. 2008) and analysis of visiting bees in plants (Marchini et al. 2001, Santos et al. 2006, Vieira et al. 2008).

The knowledge of the regional flora with beekeeping potential possibilities the better use of the floral resources and stimulate the utilization of species of plants with apiarist value in the restoration of the vegetation of rural areas.

This research was carried out to obtain a floristic survey in the surroundings areas of apiaries in rural area in the counties of Ubiratã and Nova Aurora (PR) to identify the floral sources used by Apis mellifera for honey production and to evaluate the pollen spectrum of the honey samples during the year.

\section{MATERIALS AND METHODS}

AREA OF STUDY

The collection areas (Figure 1) are located between $24^{\circ} 20^{\prime}$ to $24^{\circ} 40^{\prime} \mathrm{S}$ and $52^{\circ} 52^{\prime}$ to $53^{\circ} 26^{\prime}$ 'W parallels, distributed in three rural areas in the counties of Ubiratã in the Central-Western meso-region of Paraná State and Nova Aurora in the OccidentalCentre meso region of Paraná State.

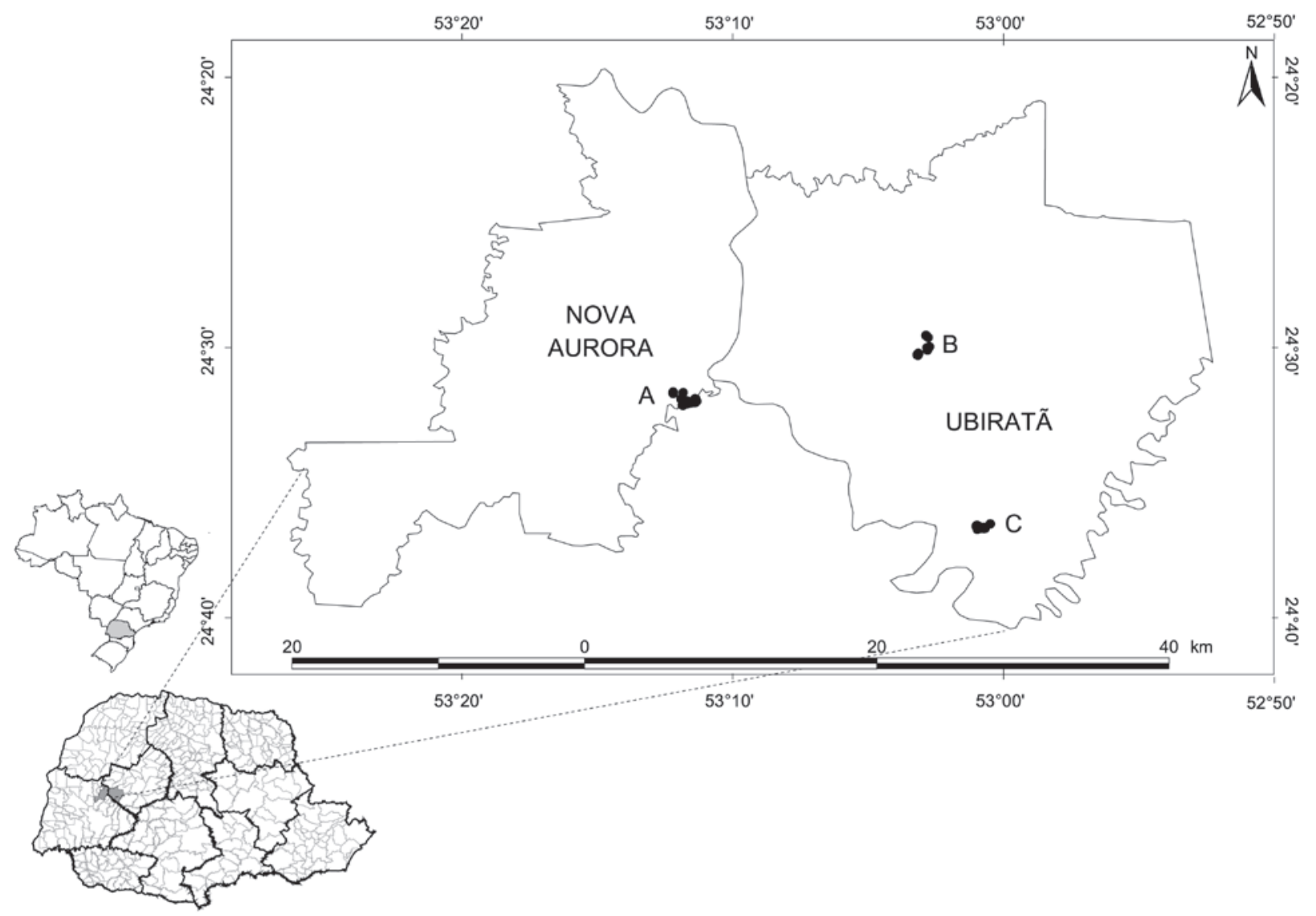

Figure 1 - Location of the collection points in the counties of Nova Aurora (Point A) and Ubiratã (Points B and C) in Paraná state. 
These counties are located in the phytogeographical domains of Submontane Semidecidous Tropical Forest (IBGE 1992). The predominant soil is Red Latosol (Brazilian classification: Latossolo Vermelho distroférrico, Embrapa 1999) and the region presents a softly waved relief, located in the third plateau of Paraná State at $550 \mathrm{~m}$ of height above sea level.

The climate in the region is $\mathrm{CwB}$, humid subtropical, with noticeable dry winters and very rainy summers by Köppen's classification. The mean annual temperature is higher than $22^{\circ} \mathrm{C}$ in the warmest month and lower than $18^{\circ} \mathrm{C}$ in the coldest months. The pluviometric precipitation varies from $1,600 \mathrm{~mm}$ to $1,900 \mathrm{~mm}$ and the relative humidity of the air remains close to $80 \%$ (IAPAR 2009).

\section{LOCATION OF THE APIARIES}

The collections were carried out in three apiaries, one in the county of Nova Aurora (2431'50" S and $53^{\circ} 11^{\prime} 50^{\prime \prime} \mathrm{W}-$ Apiary A) and two in the county of Ubiratã -PR (24⒉ $29^{\prime} 41^{\prime \prime} \mathrm{S}$ and $53^{\circ} 02^{\prime} 43^{\prime \prime} \mathrm{W}$ - Apiary B and $24^{\circ} 36^{\prime} 40.8^{\prime \prime} \mathrm{S}$ and $53^{\circ} 00^{\prime} 52.6^{\prime \prime}$ $\mathrm{W}$ - Apiary C). The sampled areas embrace forest remnants, gardens, pastures, agricultural cultivations (predominance of soybean and corn) and reforestation areas (predominance of Eucalyptus). Apiary A has a legal reserve area of 13.65 ha, Apiary B of 2.4 ha and Apiary C of 15.64 ha.

COLLECTION AND IDENTIFICATION OF THE BOTANICAL MATERIAL

The collections were carried out biweekly from October 2008 to November 2009, totalizing 13 months. Considering that the radius of the forest remnants in the surrounding area of the apiaries did not exceed 1,000 meters and the others rural properties were composed by agricultural areas, random collections were determined inside of a radius of approximately $1,000 \mathrm{~m}$ around the apiaries. The vegetation survey was conducted with usual techniques of floristic surveys (Fidalgo and Bononi 1989, IBGE 1992).
Five samples of each vegetal specie in blooming were collected and then herborized and identified (Cronquist 1981) at Herbarium of the Universidade Tecnológica Federal do Paraná (HCF) in Campo Mourão. When necessary, these samples were also sent to the Botanical Museum of Curitiba (MBM) and to the Herbarium of the Botanical Department of the Universidade Federal do Paraná (UFPR) in Curitiba. The material was recorded using a digital camera Sony-DSC-H9 (8.1MP-15x of optical zoom).

The classification of the species by the habit was adopted in accordance with HCF: tree - higher than $5 \mathrm{~m}$ of height and small tree - lower than $5 \mathrm{~m}$, both forming frustum (trunk); shrub - with woody tissue and until $5 \mathrm{~m}$ of height, without forming frustum; herb - with no woody tissue. The classification was done in successional stages (Reitz et al. 1978, 1983, SPVS 1996, Roderjan et al. 1998).

\section{Collection of Honey SAMPles}

In each apiary, three beehives were marked next to the forest remnant and to the agricultural cultures. Empty frame with beeswax foundation in the super was set up monthly to avoid the mixture of the honey produced in different months. Samples were collected from December 2008 to April 2009 and from May to November 2009 when there was honey stored in the combs, even if it was not mature (with minimum $75 \%$ of capped cells). The honeycomb was centrifuged separately in plastic packing to avoid the mixture of the different samples.

\section{Reference SLide Collection ANd Pollen ANALyses}

Floral buds were collected and packed separately to avoid the pollen mixture. One pollen slide collection was prepared using the acetolysis method (Erdtman 1952). This method is used to eliminate cell contents and enable observations on pollen size, shape, aperture and exine surface. A camera coupled in a microscope and the Motic images 2000 software captured images of the pollen. 
To the preparation of the slides of pollen contained in the honey of each month, the samples of the three beehives of each apiary were mixed. To the samples with moisture above $20 \%$, it was used a quantity proportionally higher. An aliquot of $10 \mathrm{~g}$ was diluted in $20 \mathrm{~mL}$ of distilled water and centrifuged to the acetolysis method. Two slides of each sample were prepared for posterior counting and identification of the pollen types.

Theslides were observed in a optical microscope Motic to the qualitative analyses (determination of pollen types) and quantitative (counting of pollen grains). Pollen types were determined by comparing with the reference lamina and also by researches in the specialized bibliography (Barth 1989, Moreti et al. 2002, Melhem et al. 2003, Roubik 2010). To the quantitative analyses, 300 to 500 pollen grains of each slide were counted and the averages of the counting of two slides of each sample were considered. The pollen types were classified in four classes of frequency (Louveaux et al. 1978, Barth 1989): dominant pollen (frequency higher than $45 \%$ - D), accessory pollen (16\% to $45 \%$ - A), important isolated pollen (3\% to $15 \%$ I) and occasional isolated pollen (frequency lower than $3 \%-O$ ).

The Diversity Index of Shannon (H') (Pielou 1975) and Simpson (D) (Poole 1974) were calculated to the pollen data:
$\mathrm{H}^{\prime}=-\Sigma$ pi $\mathrm{x} \ln$ pi, where: $\mathrm{pi}=\mathrm{ni} / \mathrm{N}, \mathrm{ni}=$ number of individuals of the specie $\mathrm{i}, \mathrm{N}=$ total number of sampled individuals.

$1-\mathrm{D}=1-\Sigma\{[$ ni (ni-1)]/N(N-1) $\}$, where: ni $=$ number of individuals of $\mathrm{n}$-th specie, $\mathrm{N}=$ total number of sampled individuals.

\section{RESULTS}

SURVEY OF THE PLANTS

In the floristic survey, 208 species were recorded and distributed in 66 families (Table I). Six families, Asteraceae (9.48\%), Myrtaceae (7.11\%), Solanaceae (5.69\%), Malpighiaceae (4.27\%), Bignoniaceae (5.21\%) and Fabaceae (3.79\%), concentrate approximately $36 \%$ of the richness of the area. Genera with higher richness of species were Solanum (seven species) and Eucalyptus (four species).

It can be observed in Table I that 162 species $(76.8 \%)$ are native and 34 species $(16.1 \%)$ are exotic from identified species. Among them, there were introduced species, cultivated in the areas close to the apiaries and with economical importance, such as Eucalyptus spp. and Glycine max (soybean).

From the total of the species, $139(67.0 \%)$ were classified as pioneer species and $52(25.0 \%)$ as secondary. 53 arboreal species (25.5\%), 41 herbs (19.7\%), 40 shrubs (19.2\%), 31 climbers $(15.0 \%)$, 12 sub-shrubs (5.8\%) and 12 small trees (5.8\%) were collected.

TABLE I

Blooming period of the collected species in the counties of Nova Aurora and Ubiratã (PR) from October 2008 to November 2009.

\begin{tabular}{|c|c|c|c|c|c|c|c|c|c|c|c|c|c|c|c|c|c|c|}
\hline \multirow{2}{*}{ HCF } & \multirow{2}{*}{ Family/Specie } & \multirow{2}{*}{$\begin{array}{c}\text { Ordinary } \\
\text { name }\end{array}$} & \multirow{2}{*}{$\mathbf{H b}$} & \multirow{2}{*}{$\mathbf{O}$} & \multirow{2}{*}{ SE } & \multirow{2}{*}{ B } & \multicolumn{12}{|c|}{ Months } \\
\hline & & & & & & & $\mathbf{J}$ & $\mathbf{F}$ & $\mathbf{M}$ & $\mathbf{A}$ & $\mathbf{M}$ & $\mathbf{J}$ & $\mathbf{J}$ & $\mathbf{A}$ & $\mathbf{S}$ & $\mathbf{O}$ & $\mathbf{N}$ & D \\
\hline \multicolumn{19}{|c|}{ Acanthaceae } \\
\hline 7282 & Justicia brasiliana Roth. & & B & $\mathrm{N}$ & $\mathrm{P}$ & & - & ○ & - & - & - & & & & & $\diamond$ & & \\
\hline \multicolumn{19}{|c|}{ Alstroemeriaceae } \\
\hline 7528 & Bomarea edulis (Tuss.)Herb. & & ST & $\mathrm{N}$ & $\mathrm{P}$ & & $\diamond$ & $\diamond$ & & & & & & & & $\diamond$ & & \\
\hline \multicolumn{19}{|c|}{ Amaranthaceae } \\
\hline 7820 & Alternanthera ficoidea (L.) P. Beauv. & & $\mathrm{H}$ & & . & & & & $\diamond$ & & & & & & & & & \\
\hline
\end{tabular}


TABLE I (CONTINUATION)

\begin{tabular}{|c|c|c|c|c|c|c|c|c|c|c|c|c|c|c|c|c|c|c|c|c|}
\hline \multirow{2}{*}{ HCF } & \multirow{2}{*}{ Family/Specie } & \multirow{2}{*}{ Ordinary name } & \multirow{2}{*}{$\mathbf{H b}$} & \multirow{2}{*}{$\mathbf{O}$} & \multirow{2}{*}{ SE } & \multirow{2}{*}{\multicolumn{2}{|c|}{ B }} & \multicolumn{13}{|c|}{ Months } \\
\hline & & & & & & & & $\mathbf{J}$ & $\mathbf{F}$ & $\mathbf{M}$ & $\mathbf{A}$ & $\mathbf{M}$ & $\mathbf{J}$ & \begin{tabular}{|l|l} 
& $\mathbf{J}$ \\
\end{tabular} & \begin{tabular}{|l|l} 
& $\mathbf{A}$ \\
\end{tabular} & & $\mathbf{S}$ & $\mathbf{O}$ & $\mathbf{N}$ & D \\
\hline \multicolumn{21}{|c|}{ Amaranthaceae } \\
\hline 7827 & Chamissoa altissima (Jacq.) H. B. Kunth & & $\mathrm{H}$ & & . & & & & & $\diamond$ & & & & & & & & & & \\
\hline 7919 & Chamissoa sp. & & $\mathrm{B}$ & $\mathrm{N}$ & . & & & & & & & $\diamond$ & & & & & & & & \\
\hline 7280 & Hebanthe paniculata Mart. & ginseng & $\mathrm{B}$ & $\mathrm{N}$ & $\mathrm{P}$ & & & & & & & & $\bullet$ & $\bullet$ & $\diamond$ & & • & $\diamond$ & & \\
\hline \multicolumn{21}{|c|}{ Anacardiaceae } \\
\hline 7980 & Mangifera indica $\mathrm{L}^{4}$ & mangueira & $\mathrm{T}$ & $\mathrm{E}$ & $\mathrm{P}$ & + & & & & & & $\diamond$ & & & $\bullet$ & & $\diamond$ & $\bullet$ & & \\
\hline 7754 & Schinus terebinthifolius Raddi $^{2,4,6}$ & aroeira pimenteira & $\mathrm{S}$ & $\mathrm{N}$ & $\mathrm{P}$ & + & & $\mathbf{P}$ & - & $\bullet$ & $\bullet$ & $\bullet$ & & & $\bullet$ & & & $\diamond$ & $\bullet$ & \\
\hline \multicolumn{21}{|c|}{ Apiaceae } \\
\hline 7645 & Hydrocotyle leucocephala Cham. \& Schltdl ${ }^{3,4}$ & acariçoba & $\mathrm{H}$ & $\mathrm{N}$ & S & + & & & $\diamond$ & $\bullet$ & $\bullet$ & $\bullet$ & & & & & & & & \\
\hline \multicolumn{21}{|c|}{ Apocynaceae } \\
\hline 7261 & Condylocarpon isthmicum (Vell.) A. DC. & & ST & $\mathrm{N}$ & $\mathrm{P}$ & & & & - & $\bullet$ & & & & & & & $\bullet$ & 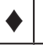 & $\bullet$ & \\
\hline 7646 & Prestonia coalita (Vell.) Woodson & Cipo-de-leite & ST & $\mathrm{N}$ & $\mathrm{P} /$ & & & & $\diamond$ & & & & & & & & & & & \\
\hline 7507 & Rauwolfia sellowii Muell. Arg. & & & & & & & & & & & & & & & & & & & \\
\hline \multirow[t]{2}{*}{7243} & Tabernaemontana catharinensis A.DC & leiteiro & $\mathrm{T}$ & $\mathrm{N}$ & $\mathrm{P}$ & & & & & & $\bullet$ & $\bullet$ & $\bullet$ & & & & $\bullet$ & $\diamond$ & $\bullet$ & \\
\hline & Asclepiadaceae & & & & & & & & & & & & & & & & & & & \\
\hline 7288 & Asclepias curassavica L. & & $\mathrm{H}$ & $\mathrm{N}$ & $\mathrm{P}$ & & & & $\diamond$ & $\diamond$ & $\diamond$ & $\bullet$ & $\bullet$ & $\bullet$ & 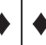 & & $\diamond$ & $\diamond$ & $\bullet$ & $\bullet$ \\
\hline \multicolumn{21}{|c|}{ Asteraceae } \\
\hline 7536 & Ageratum conyzoides L. & & $\mathrm{H}$ & $\mathrm{N}$ & S & & & 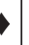 & - & $\bullet$ & $\bullet$ & $\bullet$ & $\bullet$ & & & & & & & \\
\hline 7531 & Baccharis dracunculifolia DC. ${ }^{6,8}$ & vassoura & B & $\mathrm{N}$ & $\mathrm{P}$ & - & & 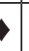 & • & $\bullet$ & $\bullet$ & $\bullet$ & & & & & & $\bullet$ & & \\
\hline 7921 & Baccharis microdonta DC. ${ }^{1,2,8}$ & vassoura & $\mathrm{B}$ & $\mathrm{N}$ & $\mathrm{P}$ & $x$ & & & & $\bullet$ & & $\diamond$ & & & & & & & & \\
\hline 7266 & Bidens pilosa $\mathrm{L} .{ }^{2,3,4}$ & picão preto & $\mathrm{H}$ & $\mathrm{N}$ & $\mathrm{P}$ & - & & & & $\bullet$ & & $\bullet$ & & & & & $\bullet$ & $\checkmark$ & $\bullet$ & \\
\hline 8168 & Calea pinnatifida (R.Br.) Less. & & SB & $\mathrm{N}$ & $\mathrm{P}$ & & & & & & & & & & & & $\diamond$ & $\bullet$ & $\bullet$ & \\
\hline 7819 & Calyptocarpus biaristatus (DC.) H. Rob. & & $\mathrm{H}$ & $\mathrm{N}$ & S & & & & & $\diamond$ & & $\bullet$ & & & & & & & $\bullet$ & $\bullet$ \\
\hline 7292 & Centratherum punctatum Cass. & & $\mathrm{H}$ & $\mathrm{N}$ & $\mathrm{P}$ & & & t & $\diamond$ & $\diamond$ & & $\diamond$ & & $\diamond$ & & & $\diamond$ & 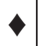 & & \\
\hline 7643 & Chromolaena pedunculosa (Hook. \& Arn.)R.M.\&H. & & $\mathrm{H}$ & $\mathrm{N}$ & $\mathrm{P}$ & & & & $\bullet$ & $\bullet$ & $\bullet$ & $\bullet$ & & & & & & & & \\
\hline 7523 & Conyza bonariensis (L.) Cronquist $^{6}$ & voadeira & $\mathrm{H}$ & $\mathrm{N}$ & $\mathrm{P}$ & $x$ & & 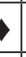 & $\bullet$ & & & & $\bullet$ & & & & & & $\bullet$ & \\
\hline 7920 & Dasyphyllum brasiliense (Spr.) Cabr. & sucará & SB & $\mathrm{N}$ & $\mathrm{S}$ & & & & & $\bullet$ & $\checkmark$ & $\checkmark$ & $\checkmark$ & & $\bullet$ & & $\bullet$ & & & \\
\hline 7291 & Elephantopus mollis Kunth ${ }^{6}$ & sarsuiá & $\mathrm{H}$ & $\mathrm{N}$ & $\mathrm{P}$ & $x$ & & & $\diamond$ & $\bullet$ & $\bullet$ & $\bullet$ & $\bullet$ & $\bullet$ & & & & 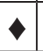 & & \\
\hline 7267 & Emilia sonchifolia $\left(\right.$ L.) DC. ${ }^{2,3,4}$ & emilia & $\mathrm{H}$ & $\mathrm{N}$ & $\mathrm{P}$ & - & & & - & & & & & & $\bullet$ & & & 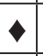 & • & \\
\hline 8173 & Galinsoga parviflora Cav. & & $\mathrm{H}$ & $\mathrm{N}$ & $\mathrm{P}$ & & & & & & & & & & & & $\diamond$ & & & \\
\hline 8109 & Mikania cordifolia (L.f)Willd. $^{4}$ & cipó-guaco & ST & $\mathrm{N}$ & $\mathrm{P}$ & - & & & & & $\bullet$ & & & & $\diamond$ & & & & & \\
\hline 7752 & Mikania micrantha Kunth $^{9}$ & cipó-guaco & ST & $\mathrm{N}$ & $\mathrm{P} / \mathrm{s}$ & $x$ & & & $\diamond$ & $\checkmark$ & $\bullet$ & $\bullet$ & & & & & & & & \\
\hline 7651 & Parthenium hysterophorus $\mathrm{L}^{3}$ & losna-branca & $\mathrm{H}$ & $\mathrm{N}$ & $\mathrm{P}$ & - & & & $\bullet$ & & $\bullet$ & $\bullet$ & & 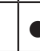 & $\bullet$ & & & & & \\
\hline 7247 & Senecio brasiliensis (Spreng.) Less. ${ }^{2,4,6,7}$ & berneira & $\mathrm{H}$ & $\mathrm{N}$ & $\mathrm{P}$ & - & & & & & & & & 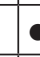 & • & & $\bullet$ & $\bullet$ & $\bullet$ & \\
\hline 8112 & Sonchus asper (L.)Hill. & Serralha-brava & $\mathrm{H}$ & $\mathrm{N}$ & $\mathrm{P}$ & & & & & & & & & & 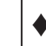 & & & & & \\
\hline 8111 & Sonchus oleraceus $\mathrm{L}^{4}$ & serralha & $\mathrm{H}$ & $\mathrm{N}$ & $\mathrm{P}$ & - & & & & $\bullet$ & & & & 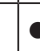 & , & & - & & & \\
\hline 7278 & Wedelia subvelutina DC. & & $\mathrm{H}$ & $\mathrm{N}$ & $\mathrm{P}$ & & & & & & & & & & & & & $\bullet$ & & \\
\hline \multicolumn{21}{|c|}{ Begoniaceae } \\
\hline 7390 & Begonia cucullata Willd. & azedinha & $\mathrm{H}$ & $\mathrm{N}$ & $\mathrm{S}$ & & & 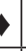 & $\diamond$ & $\bullet$ & & & & & & & $\diamond$ & - & $\bullet$ & $\bullet$ \\
\hline 7242 & Adenocalymma marginatum (Cham.) DC. & & $\mathrm{CT}$ & $\mathrm{N}$ & $\mathrm{P}$ & & & & $\bullet$ & $\bullet$ & & & & & & & & $\diamond$ & & \\
\hline 7548 & Arrabidaea chica (Humb. \& Bonpl.) B.Verlot & & $\mathrm{CT}$ & $\mathrm{N}$ & $\mathrm{P}$ & & & 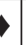 & & & $\bullet$ & & & & & & • & $\bullet$ & & \\
\hline
\end{tabular}


TABLE I (CONTINUATION)

\begin{tabular}{|c|c|c|c|c|c|c|c|c|c|c|c|c|c|c|c|c|c|c|c|}
\hline \multirow{2}{*}{ HCF } & \multirow{2}{*}{ Family/Specie } & \multirow{2}{*}{ Ordinary name } & \multirow{2}{*}{$\mathbf{H b}$} & \multirow{2}{*}{$\mathbf{O}$} & \multirow{2}{*}{ SE } & \multirow{2}{*}{\multicolumn{2}{|c|}{ B }} & \multicolumn{12}{|c|}{ Months } \\
\hline & & & & & & & & $\mathbf{J}$ & $\mathbf{F}$ & $\mathbf{M}$ & $\mathbf{A}$ & $\mathbf{M}$ & $\mathbf{J}$ & $\mathbf{J}$ & $\mathbf{A}$ & $\mathbf{S}$ & $\mathbf{O}$ & $\mathbf{N}$ & $\mathbf{D}$ \\
\hline \multicolumn{20}{|c|}{ B.Verlot } \\
\hline 7505 & Arrabidaea selloi (Spreng.) Sandw. & & $\mathrm{CT}$ & $\mathrm{N}$ & $\mathrm{P}$ & & & 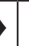 & & & & & & & & & & & $\diamond$ \\
\hline 8172 & Clytostoma sciuripabulum Bareau \& K.Schum & & CT & $\mathrm{N}$ & $\mathrm{P}$ & & & & & & & & 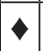 & & & & & & \\
\hline 8169 & 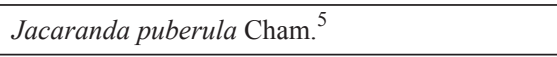 & caroba & $\mathrm{T}$ & $\mathrm{N}$ & $\mathrm{P}$ & $\mathrm{x}$ & & & & & & & & & & $\diamond$ & & & \\
\hline 7549 & Macfadyena unguis-cati (L.) A. H. Gentry & unha-de-gato & CT & $\mathrm{N}$ & $\mathrm{P}$ & & & 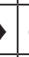 & - & $\bullet$ & $\diamond$ & & & & $\bullet$ & $\bullet$ & $\bullet$ & $\bullet$ & \\
\hline 7391 & Mansoa difficilis (Cham.) Bureau. \& K.Schum. ${ }^{8}$ & mansoa & CT & $\mathrm{N}$ & $\mathrm{S}$ & $\mathrm{x}$ & & & & & & & & - & $\bullet$ & $\bullet$ & $\bullet$ & $\bullet$ & \\
\hline 7276 & Pyrostegia venusta (Ker Grawl) Miers ${ }^{4,6,9}$ & cipó-de-são-joão & CT & $\mathrm{N}$ & $\mathrm{P}$ & + & & & & & $\diamond$ & $\diamond$ & & $\diamond$ & $\diamond$ & $\diamond$ & $\diamond$ & $\diamond$ & \\
\hline 8211 & Tecoma stans (L.)Adr. Juss. ex Kunth & & $\mathrm{B}$ & $\mathrm{E}$ & $\mathrm{P}$ & & & & & & & & $\bullet$ & & & & $\diamond$ & & \\
\hline 7253 & Tynanthus micranthus Mello & & ST & $\mathrm{N}$ & $\mathrm{P} / \mathrm{S}$ & & & & & & & & & & & - & $\bullet$ & & \\
\hline \multicolumn{20}{|c|}{ Bixaceae } \\
\hline 7530 & Bixa orelana $\mathrm{L}^{5}$ & urucum & $\mathrm{T}$ & $\mathrm{N}$ & $\mathrm{P}$ & + & & 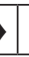 & & $\bullet$ & & $\bullet$ & & & $\bullet$ & & & & \\
\hline \multicolumn{20}{|c|}{ Boraginaceae } \\
\hline 7527 & Cordia ecalyculata Vell. & Café de bugre & S & $\mathrm{N}$ & $\mathrm{P}$ & & & 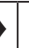 & & & $\bullet$ & & & & & & $\bullet$ & & \\
\hline 7751 & Cordia trichotoma (Vell.) Arrab. ex Steud. ${ }^{4}$ & óleo-pardo, louro-pardo & $\mathrm{T}$ & $\mathrm{N}$ & $\mathrm{P}$ & + & & & $\bullet$ & $\diamond$ & $\bullet$ & & & $\bullet$ & & & & & \\
\hline 7251 & Heliotropium transalpinium Vell. & & $\mathrm{H}$ & $\mathrm{N}$ & $\mathrm{P}$ & & & 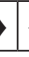 & 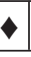 & $\diamond$ & $\diamond$ & & 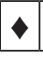 & 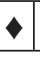 & $\bullet$ & $\bullet$ & $\diamond$ & $\diamond$ & \\
\hline \multicolumn{20}{|c|}{ Brassicaceae } \\
\hline 8059 & Brassica napus L. & & $\mathrm{H}$ & & . & & & & & & & & & & $\diamond$ & & & & \\
\hline 8064 & Raphanus sativus L. & & $\mathrm{H}$ & & · & & & & & & & & & & $\diamond$ & & & & \\
\hline \multicolumn{20}{|c|}{ Cactaceae } \\
\hline 7400 & Brasiliopuntia brasiliensis (Willd.) Berg. & cacto & SB & $\mathrm{N}$ & $\mathrm{P}$ & & & $\Rightarrow$ & & & $\bullet$ & & & & & & & $\diamond$ & \\
\hline 7644 & Pereskia aculeata Mill. & ora-pronobilis & SB & $\mathrm{N}$ & $\mathrm{P} / \mathrm{s}$ & & & & $\checkmark$ & $\bullet$ & & & & & $\bullet$ & & & & \\
\hline 8105 & Rhipsalis cereuscula (How)Volguin & Rabo-de-rato & EPI & $\mathrm{N}$ & $\mathrm{S}$ & & & & & & $\bullet$ & & $\bullet$ & & $\diamond$ & $\bullet$ & $\bullet$ & - & \\
\hline \multicolumn{20}{|c|}{ Caesalpinaceae } \\
\hline 7521 & Bauhinia forficata Link $^{2,4,5,8}$ & pata-de-vaca & $\mathrm{S}$ & $\mathrm{N}$ & $\mathrm{P}$ & $\mathrm{x}$ & & 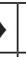 & $\checkmark$ & $\bullet$ & $\bullet$ & $\bullet$ & & & & & - & & \\
\hline 7750 & Phanera microstachya (Raddi) L.P. & & CT & $\mathrm{N}$ & . & $\mathrm{x}$ & & & & $\diamond$ & & & & & & & & & \\
\hline 8217 & Caesalpinia peltophoroides Benth. ${ }^{1,2,3,4,5,8}$ & sibipiruna & $\mathrm{T}$ & $\mathrm{N}$ & $\mathrm{P}$ & + & & & $\bullet$ & & & $\bullet$ & & & & & $\bullet$ & - & \\
\hline 7822 & Senna neglecta (Vogel) Irwin \& Barneby ${ }^{4,5}$ & chuva-de-ouro & $\mathrm{B}$ & $\mathrm{N}$ & $\mathrm{P}$ & $\mathrm{x}$ & & & & $\diamond$ & & & & & & & & & \\
\hline \multicolumn{20}{|c|}{ Caricaceae } \\
\hline \multirow[t]{2}{*}{7525} & Carica papaya $\mathrm{L}^{4}$ & mamoeiro & $\mathrm{T}$ & $\mathrm{E}$ & $\mathrm{P}$ & + & & 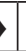 & $\checkmark$ & $\diamond$ & $\diamond$ & & & $\diamond$ & $\bullet$ & $\bullet$ & & & \\
\hline & Cecropiaceae & & & & & & & & & & & & & & & & & & \\
\hline 7509 & Cecropia pachystachya Trécul. 8 & embaúba & $\mathrm{T}$ & $\mathrm{N}$ & $\mathrm{P}$ & $\mathrm{x}$ & & D & & & & & $\bullet$ & & - & & - & & $\diamond$ \\
\hline \multicolumn{20}{|c|}{ Clusiaceae } \\
\hline 7399 & Clusia sp. & & $\mathrm{B}$ & $\mathrm{E}$ & $\mathrm{P}$ & & & & & & & & & & & & & $\diamond$ & \\
\hline & Combretaceae & & & & & & & & & & & & & & & & & & \\
\hline 7538 & Combretum fruticosum (Loef.) Stunz & escova-de-macaco & $\mathrm{TE}$ & $\mathrm{N}$ & $\mathrm{P}$ & & & 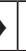 & $\bullet$ & $\bullet$ & & & & & & & & & \\
\hline & Combretaceae & & & & & & & & & & & & & & & & & & \\
\hline 8163 & Terminalia australis Cambess ${ }^{4}$ & amarilho, sarandi & $\mathrm{T}$ & $\mathrm{N}$ & $\mathrm{S}$ & $\mathrm{x}$ & & & & & & & & & $\diamond$ & $\bullet$ & $\bullet$ & & \\
\hline & Commelinaceae & & & & & & & & & & & & & & & & & & \\
\hline 7534 & Dichorisandra hexandra (Aubl.) Standl & & $\mathrm{H}$ & $\mathrm{N}$ & $\mathrm{Sl}$ & & & 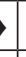 & • & $\bullet$ & - & & & & & & & & $\bullet$ \\
\hline 7641 & Tripogandra diuretica (Mart.) Handlos & & $\mathrm{H}$ & $\mathrm{N}$ & $\mathrm{P} / \mathrm{s}$ & & & & $\diamond$ & $\checkmark$ & $\bullet$ & & & - & & & & & \\
\hline & Convolvulaceae & & & & & & & & & & & & & & & & & & \\
\hline 7826 & Ipomoea nil (L.)Roth $^{4}$ & corda-de-viola & ST & $\mathrm{N}$ & $\mathrm{P}$ & $x$ & & & & $\diamond$ & & & & - & & & & & \\
\hline
\end{tabular}


TABLE I (CONTINUATION)

\begin{tabular}{|c|c|c|c|c|c|c|c|c|c|c|c|c|c|c|c|c|c|c|c|}
\hline \multirow{2}{*}{ HCF } & \multirow{2}{*}{ Family/Specie } & \multirow{2}{*}{ Ordinary name } & \multirow{2}{*}{$\mathbf{H b}$} & \multirow{2}{*}{$\mathbf{O}$} & \multirow{2}{*}{ SE } & \multirow{2}{*}{ B } & \multicolumn{13}{|c|}{ Months } \\
\hline & & & & & & & J & & $\mathbf{F}$ & $\mathbf{M}$ & $\mathbf{A}$ & $\mathbf{M}$ & $\mathbf{J}$ & $\mathbf{J}$ & $\mathbf{A}$ & $\mathbf{S}$ & $\mathbf{O}$ & $\mathbf{N}$ & D \\
\hline \multicolumn{20}{|c|}{ Convolvulaceae } \\
\hline 7647 & Ipomoea quamoclit $\mathrm{L}^{4}$ & esqueleto & ST & $\mathrm{N}$ & $\mathrm{P}$ & $\mathrm{x}$ & & & $\diamond$ & & & & $\bullet$ & & & & & & \\
\hline \multicolumn{20}{|c|}{ Cucurbitaceae } \\
\hline 7376 & Momordica charatia $\mathrm{L}^{4}$ & & CT & E & $\mathrm{P}$ & + & $\checkmark$ & & • & $\diamond$ & & $\bullet$ & & $\bullet$ & & & & 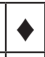 & \\
\hline 7260 & Wilbrandia longisepala Cogn. & & CT & $\mathrm{N}$ & $\mathrm{P}$ & & 8 & & $\bullet$ & $\bullet$ & & & & & & $\bullet$ & $\diamond$ & & \\
\hline \multicolumn{20}{|c|}{ Cyperaceae } \\
\hline \multirow[t]{2}{*}{7386} & Cyperus luzulae (L.) Retz & & & $\mathrm{N}$ & $\mathrm{P}$ & & & & & & $\bullet$ & & & & & & & $\bullet$ & \\
\hline & \multicolumn{19}{|l|}{ Ebenaceae } \\
\hline 8219 & Diospyrus kaki L.f. & caqui & & $\mathrm{E}$ & $\mathrm{P}$ & & & & & & & & & & & & $\diamond$ & $\bullet$ & \\
\hline \multicolumn{20}{|c|}{ Ericaceae } \\
\hline 7373 & Rhododendron simsii Planch. $^{4}$ & azaléia & B & $\mathrm{E}$ & $\mathrm{S}$ & + & & & & & $\bullet$ & & $\bullet$ & & & & & 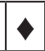 & \\
\hline & \multicolumn{19}{|l|}{ Euphorbiaceae } \\
\hline 8214 & Alcalypha gracilis Spreng. ${ }^{3}$ & tapa-buraco & $\mathrm{H}$ & $\mathrm{N}$ & $\mathrm{S}$ & $\mathrm{x}$ & & & $\bullet$ & & - & $\bullet$ & & & & $\bullet$ & $\diamond$ & $\bullet$ & $\bullet$ \\
\hline 7924 & Alchornea iricurana Cass. $^{8}$ & fruta-de-pombo & $\mathrm{T}$ & $\mathrm{N}$ & $\mathrm{P}$ & $\mathrm{x}$ & & & & & & 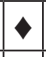 & & & $\bullet$ & & & $\bullet$ & \\
\hline 7252 & Alchornea triplinervia (Spreng.) M. Arg. ${ }^{8}$ & tapiá, tanheiro & $\mathrm{T}$ & $\mathrm{N}$ & $\mathrm{P}$ & $\mathrm{x}$ & & & & & $\bullet$ & $\bullet$ & $\bullet$ & & $\bullet$ & $\bullet$ & $\bullet$ & $\bullet$ & \\
\hline 7650 & Bernardia pulchella (Baill.) Muell. Arg. & & $\mathrm{B}$ & $\mathrm{N}$ & $\mathrm{S}$ & & & & $\diamond$ & & & & & $\bullet$ & & $\bullet$ & & $\bullet$ & $\bullet$ \\
\hline 7285 & Dalechampia stipulacea Muell. Arg. & Cipó-urtiga & ST & $\mathrm{N}$ & $P$ & . & & & & & & & & & & & $\diamond$ & & \\
\hline 7979 & Manihot esculenta Crantz. $^{6}$ & mandioca & B & $\mathrm{N}$ & $\mathrm{P}$ & + & & & & & & $\diamond$ & & & & & & & \\
\hline 7262 & Ricinus communis L. ${ }^{2,5,6,8}$ & mamona & $\mathrm{S}$ & E & $\mathrm{P}$ & + & & & & $\bullet$ & $\checkmark$ & & $\checkmark$ & $\bullet$ & $\bullet$ & $\bullet$ & $\bullet$ & & 1 \\
\hline 7992 & Sebastiania brasiliensis Spreng. & leiteiro, branquilho & $\mathrm{S}$ & $\mathrm{N}$ & $\mathrm{S}$ & $\mathrm{x}$ & & & & $\bullet$ & $\diamond$ & & $\diamond$ & & & $\bullet$ & & $\bullet$ & \\
\hline \multicolumn{20}{|c|}{ Fabaceae } \\
\hline 7504 & Dalbergia frutescens (Vell.) Britton. & cipó-rabo-de-bugio & & $\mathrm{N}$ & $\mathrm{P} / \mathrm{S}$ & & & & $\bullet$ & $\bullet$ & & & & & & & & $\bullet$ & $\checkmark$ \\
\hline 7392 & Desmodium incanum DC. ${ }^{1,2,3}$ & carrapicho & & $\mathrm{N}$ & $\mathrm{P}$ & $\mathrm{x}$ & & & & & & & & & & & 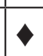 & $\diamond$ & \\
\hline 7384 & Glycine $\max (\mathrm{L}$.$) Merr. ^{4}$ & Soja & & $\mathrm{E}$ & $\mathrm{P}$ & + & & & & $\diamond$ & & & & & & & & $\checkmark$ & $\bullet$ \\
\hline 7506 & Lonchocarpus campestris Mart. ex Benth. & paufarinha & $\mathrm{T}$ & $\mathrm{N}$ & $\mathrm{P}$ & & $\checkmark$ & & - & & $\bullet$ & & & & & & & & $\checkmark$ \\
\hline 7378 & Lonchocarpus subglaucescens Benth. & & & $\mathrm{N}$ & $\mathrm{Sl}$ & & & & & & & & & & & & & $\bullet$ & \\
\hline 7818 & Machaerium stipitatum (DC.) Vogel ${ }^{8}$ & sapuva & & $\mathrm{N}$ & $\mathrm{P} / \mathrm{S}$ & $\mathrm{x}$ & & & & 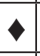 & & & & & & & & & \\
\hline 8171 & Myrocarpus frondosus Fr. Allem. & & $\mathrm{T}$ & $\mathrm{N}$ & $\mathrm{S}$ & & & & & & & & & & & $\diamond$ & $\bullet$ & & \\
\hline 7749 & Vigna venusta (Piper) Maréchal \& al. & & ST & & $\mathrm{P}$ & & & & & $\diamond$ & & & & & & & & & \\
\hline \multicolumn{20}{|c|}{ Flacourtiaceae } \\
\hline 7283 & Banara tomentosa Clos. & & $\mathrm{T}$ & $\mathrm{N}$ & $\mathrm{S} 1$ & & & & & & & & & & $\diamond$ & $\bullet$ & $\bullet$ & $\bullet$ & 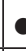 \\
\hline 8060 & Casearia sylvestris $\mathrm{Sw}$. & cafezeiro-bravo & $\mathrm{T}$ & $\mathrm{N}$ & $\mathrm{P}$ & & & & & & & & & $\bullet$ & $\diamond$ & $\diamond$ & $\bullet$ & $\bullet$ & \\
\hline 7254 & Prockia crucis P. Browne ex L. & & $\mathrm{T}$ & $\mathrm{N}$ & $\mathrm{S}$ & & & & - & & & & & & & & 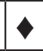 & $\bullet$ & \\
\hline & Iridaceae & & & & & & & & & & & & & & & & & & \\
\hline 7289 & Sisyrinchium luzula Klotzsch & & $\mathrm{H}$ & $\mathrm{N}$ & $\mathrm{P}$ & & & & & & & & & & & $\bullet$ & $\diamond$ & $\bullet$ & \\
\hline & Lamiaceae & & & & & & & & & & & & & & & & & & \\
\hline 7245 & Leonurus sibirucus L. ${ }^{4}$ & rubi & $\mathrm{H}$ & $\mathrm{E}$ & $\mathrm{P}$ & $\mathrm{x}$ & & & $\bullet$ & $\bullet$ & $\diamond$ & & $\bullet$ & & & $\diamond$ & 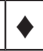 & $\bullet$ & \\
\hline 7821 & Hyptis mutabilis (L.C.Rich) Briq. ${ }^{6,8}$ & boldo-branco & $\mathrm{H}$ & $\mathrm{N}$ & $\mathrm{P}$ & $\mathrm{x}$ & & & $\checkmark$ & 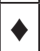 & $\bullet$ & & & & & & & & \\
\hline & Lauraceae & & & & & & & & & & & & & & & & & & \\
\hline 7397 & Nectandra lanceolata Nees \& Mart. ex Nees & canela-amarela & $\mathrm{T}$ & $\mathrm{N}$ & $\mathrm{S}$ & & & & & & & & & & $\bullet$ & $\bullet$ & $\bullet$ & 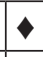 & 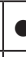 \\
\hline 8065 & Nectandra megapotamica (Spr.) Mez & & $\mathrm{T}$ & & . & & & & & & & & & & $\diamond$ & & & & \\
\hline 7918 & Ocotea puberula (Rich.) Nees & & $\mathrm{T}$ & $\mathrm{N}$ & $\mathrm{P}$ & & 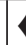 & & - & & $\bullet$ & $\diamond$ & & - & $\bullet$ & $\bullet$ & & $\bullet$ & \\
\hline
\end{tabular}




\section{TABLE I (CONTINUATION)}

\begin{tabular}{|c|c|c|c|c|c|c|c|c|c|c|c|c|c|c|c|c|c|c|}
\hline \multirow{2}{*}{ HCF } & \multirow{2}{*}{ Family/Specie } & \multirow{2}{*}{$\begin{array}{l}\text { Ordinary } \\
\text { name }\end{array}$} & \multirow{2}{*}{$\mathbf{H b}$} & \multirow{2}{*}{$\mathbf{O}$} & \multirow{2}{*}{ SE } & \multirow{2}{*}{ B } & \multicolumn{12}{|c|}{ Months } \\
\hline & & & & & & & $\mathbf{J}$ & $\mathbf{F}$ & $\mathbf{M}$ & $\mathbf{A}$ & $\mathbf{M}$ & $\mathbf{J}$ & $\mathbf{J}$ & $\mathbf{A}$ & $\mathbf{S}$ & $\mathbf{O}$ & $\mathbf{N}$ & D \\
\hline 8110 & Persea americana Mill. ${ }^{4,6,8}$ & abacateiro & $\mathrm{T}$ & E & $\mathrm{P}$ & + & & & & & & & & $\diamond$ & $\diamond$ & & & \\
\hline \multicolumn{19}{|c|}{ Liliaceae } \\
\hline 7916 & Asparagus setaceus (Kunth.) Jessop. & $\begin{array}{l}\text { aspargo- } \\
\text { ornamental }\end{array}$ & ST & $\mathrm{E}$ & $P$ & & & & & $\diamond$ & & $\bullet$ & & & & & & \\
\hline \multicolumn{19}{|c|}{ Loganiaceae } \\
\hline 7290 & Strychnos brasiliensis (Spr.) Mart. & anzol-de-lontra & $\mathrm{B}$ & $\mathrm{N}$ & $\mathrm{P} / \mathrm{Si}$ & & & $\bullet$ & $\bullet$ & $\bullet$ & & & $\bullet$ & & & $\diamond$ & $\bullet$ & \\
\hline \multicolumn{19}{|c|}{ Lythraceae } \\
\hline 7377 & Lagerstroemia indica $\mathrm{L}$. & Resedá & $\mathrm{T}$ & $\mathrm{E}$ & $\mathrm{P}$ & & $\diamond$ & & $\bullet$ & & & & & & & & $\diamond$ & $\bullet$ \\
\hline 7372 & Punica granatum $\mathrm{L}$. & romã & $\mathrm{B}$ & $\mathrm{E}$ & $\mathrm{P}$ & & & & & & & & & $\bullet$ & $\diamond$ & & $\diamond$ & $\bullet$ \\
\hline \multicolumn{19}{|c|}{ Malpighiaceae } \\
\hline 7526 & $\begin{array}{l}\text { Banisteriopsis adenopoda (Adr.Juss) } \\
\text { B.Gates }\end{array}$ & & SB & $\mathrm{N}$ & $\mathrm{P}$ & & $\diamond$ & & & & & & & & & & & \\
\hline 7522 & Banisteriopsis parviflora (Adr.Juss.) Gates ${ }^{6}$ & & SB & $\mathrm{N}$ & $\mathrm{P}$ & $\mathrm{x}$ & $\diamond$ & & & & & & & & & & & \\
\hline 7503 & Dicella nucifera Chodat & & SB & $\mathrm{N}$ & $P$ & & $\diamond$ & $\diamond$ & $\bullet$ & $\bullet$ & & & & & & & & $\diamond$ \\
\hline 7533 & Heteropterys intermedia (Adr. Juss.) Griseb & & ST & $\mathrm{N}$ & $\mathrm{P}$ & & $\diamond$ & & $\bullet$ & & $\bullet$ & & & & & & & $\bullet$ \\
\hline 7244 & Mascagnia ovatifolia (Kunth.) Griseb. & & & & . & & & & & & & & & & & $\diamond$ & & \\
\hline 7540 & Stigmaphyllon jatrophifolium Adr. Juss. & & ST & $\mathrm{N}$ & $P$ & & $\diamond$ & & & & & & & & & & & \\
\hline 7753 & Indeterminada sp. $^{5}$ & & & & . & & & & $\diamond$ & & & & & & & & & \\
\hline \multicolumn{19}{|c|}{ Malvaceae } \\
\hline 7375 & Abutilon striatum Dicks ex Lindl. & $\begin{array}{l}\text { lanterna- } \\
\text { japonesa }\end{array}$ & $\mathrm{B}$ & $\mathrm{N}$ & $P$ & & & $\diamond$ & & & & $\diamond$ & & & $\diamond$ & & $\diamond$ & \\
\hline 7978 & $\begin{array}{l}\text { Bastardiopsis densiflora (Hook. \& Arn.) } \\
\text { Hassler }\end{array}$ & & $\mathrm{T}$ & $\mathrm{N}$ & $\mathrm{P}$ & & & & & & $\diamond$ & $\bullet$ & $\bullet$ & $\bullet$ & & & & \\
\hline 7264 & Malvastrum coromandeliam (L.) Garcke ${ }^{4}$ & guanxuma & SB & $\mathrm{N}$ & $\mathrm{P}$ & + & & & & & & & & $\bullet$ & $\bullet$ & $\diamond$ & & \\
\hline 7287 & Sida rhombifolia L. $^{3,6}$ & guanxuma & $\mathrm{H}$ & $\mathrm{N}$ & $\mathrm{P}$ & + & & $\bullet$ & & $\bullet$ & & & & & & $\diamond$ & $\bullet$ & \\
\hline 7381 & Wissadula subpeltata $(\mathrm{OK})$ R.E.Fries $^{4}$ & malva-estrela & $\mathrm{B}$ & $\mathrm{N}$ & $\mathrm{P}$ & + & & 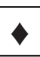 & $\diamond$ & $\diamond$ & & & & & & & 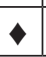 & \\
\hline \multicolumn{19}{|c|}{ Marantaceae } \\
\hline 7539 & Maranta sobolifera L. Anderss. & & $\mathrm{H}$ & $\mathrm{N}$ & $\mathrm{Si}$ & & $\diamond$ & $\checkmark$ & & & & & & & & & & \\
\hline \multicolumn{19}{|c|}{ Melastomataceae } \\
\hline 7274 & Ossaea amygdaloides (DC.) Triana & & $\mathrm{B}$ & $\mathrm{N}$ & . & & & & & & & & & & & $\diamond$ & & \\
\hline \multicolumn{19}{|c|}{ Meliaceae } \\
\hline 8213 & Cabralea canjarana (Vell.) Mart. & canjarana & $\mathrm{T}$ & $\mathrm{N}$ & $\mathrm{P}$ & & $\bullet$ & & & & & $\bullet$ & & $\bullet$ & $\diamond$ & $\diamond$ & - & \\
\hline 8113 & Melia azedarach $\mathrm{L}$. & santa-bárbara & $\mathrm{T}$ & E & $\mathrm{P}$ & & & $\bullet$ & $\bullet$ & & & & & $\diamond$ & $\diamond$ & & - & \\
\hline 7257 & Trichilia elegans A.Juss & & $\mathrm{B}$ & $\mathrm{N}$ & $S$ & & & & & & & & & & & $\diamond$ & & \\
\hline \multicolumn{19}{|c|}{ Meliaceae } \\
\hline 8004 & Trichilia pallida Swartz. & & $\mathrm{B}$ & $\mathrm{N}$ & $\mathrm{S}$ & & & & & & & & $\diamond$ & $\diamond$ & & & & \\
\hline \multicolumn{19}{|c|}{ Mimosaceae } \\
\hline 8164 & Calliandra foliolosa Benth. $^{4}$ & esponjinha & S & $\mathrm{N}$ & $\mathrm{S}$ & + & $\bullet$ & & & & & $\bullet$ & & $\bullet$ & $\diamond$ & $\bullet$ & & \\
\hline 7265 & $\begin{array}{l}\text { Enterolobium contorstisiliquum (Vell.) } \\
\text { Morong }\end{array}$ & orelha-de-nego, & $\mathrm{T}$ & $\mathrm{N}$ & $\mathrm{Si}$ & + & & & & & & & & & $\bullet$ & $\bullet$ & & \\
\hline 7277 & Leucaena leucocephala (Lam.) de Wit. ${ }^{1,2,3,4}$ & leocena & S & $\mathrm{E}$ & $P$ & + & $\bullet$ & & $\diamond$ & & & & & & & $\diamond$ & - & \\
\hline 7394 & Mimosa pudica L. ${ }^{1,4,6,8}$ & dormideira & $\mathrm{T}$ & $\mathrm{N}$ & $\mathrm{P}$ & + & & & & & & & & & & & $\bullet$ & \\
\hline 7268 & Parapiptadenia rigida (Benth.) Brenan & $\begin{array}{l}\text { angico- } \\
\text { vermelho }\end{array}$ & $\mathrm{T}$ & $\mathrm{N}$ & $P$ & & & $\bullet$ & $\bullet$ & $\bullet$ & $\bullet$ & & & & & $\diamond$ & $\bullet$ & \\
\hline
\end{tabular}


TABLE I (CONTINUATION)

\begin{tabular}{|c|c|c|c|c|c|c|c|c|c|c|c|c|c|c|c|c|c|c|c|}
\hline \multirow{2}{*}{ HCF } & \multirow{2}{*}{ Family/Specie } & \multirow{2}{*}{ Ordinary name } & \multirow{2}{*}{$\mathbf{H b}$} & \multirow{2}{*}{\multicolumn{2}{|c|}{$\mathbf{O}$}} & \multirow{2}{*}{ SE } & \multirow{2}{*}{ B } & \multicolumn{12}{|c|}{ Months } \\
\hline & & & & & & & & $\mathbf{J}$ & $\mathbf{F}$ & $\mathbf{M}$ & $\mathbf{A}$ & $\mathbf{M}$ & $\mathbf{J}$ & $\mathbf{J}$ & $\mathbf{A}$ & $\mathbf{S}$ & $\mathbf{O}$ & $\mathbf{N}$ & D \\
\hline \multicolumn{20}{|c|}{ Moraceae } \\
\hline 7284 & Maclura tinctoria (L.) D.Don ex Steud. & amora-branca & $\mathrm{T}$ & $\mathrm{N}$ & & $S$ & & & $\bullet$ & & & & & • & & $\bullet$ & $\diamond$ & • & \\
\hline \multicolumn{20}{|c|}{ Myrtaceae } \\
\hline 7990 & Calycorectes psidiiflorus (O.Berg) Sobral & & B & $\mathrm{N}$ & & $\mathrm{ii}$ & & & & & 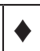 & & $\checkmark$ & & & & & 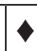 & \\
\hline 7250 & Campomanesia guazumifolia (Camb.) O. Berg. & sete-capotes & $\mathrm{T}$ & $\mathrm{N}$ & & $P$ & & & & & & & & & & & $\diamond$ & & \\
\hline 7371 & Campomanesia xanthocarpa $\mathrm{O}$ Berg. & guabiroba & $\mathrm{T}$ & $\mathrm{N}$ & & $S$ & & & & & & & & & 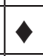 & $\bullet$ & & $\bullet$ & \\
\hline 7993 & Casearia gossypiosperma Briq (Salicaceae) & & & & & . & & & & & & & $\checkmark$ & & & & & & \\
\hline \multicolumn{20}{|c|}{ (Salicaceae) } \\
\hline 7828 & Eucalyptus saligna Smith. ${ }^{3,4,6,8,9}$ & eucalipto & $\mathrm{T}$ & $\mathrm{E}$ & & $P$ & + & & & $\bullet$ & 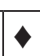 & & & & & & & & \\
\hline 7529 & Eucalyptus viminalis Labill., $3,4,6,8,9$ & eucalipto & $\mathrm{T}$ & $\mathrm{E}$ & & $P$ & $\mathrm{x}$ & $\diamond$ & & & & & & & & & & & \\
\hline 7547 & Eucalyptus sp. ${ }^{13,4,6,8,9}$ & eucalipto & $\mathrm{T}$ & $\mathrm{E}$ & & $P$ & $\mathrm{x}$ & $\diamond$ & & & & & & & & & & & \\
\hline 7395 & Eucalyptus sp. ${ }^{23,4,6,8,9}$ & eucalipto & $\mathrm{T}$ & $\mathrm{E}$ & & $P$ & $\mathrm{x}$ & & & & & & & & & & & • & \\
\hline 7914 & Eugenia hiemalis Cambess. $^{4}$ & jabuticabeira & $\mathrm{T}$ & $\mathrm{N}$ & & $P$ & $\mathrm{x}$ & & & $\bullet$ & $\diamond$ & & $\bullet$ & & $\bullet$ & & & & \\
\hline 8099 & Eugenia $\mathrm{sp}^{2,4,5}$ & & & $\mathrm{~N}$ & & $P$ & $\mathrm{x}$ & & & & & & & & 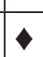 & & & & \\
\hline 7270 & Hexaclamys edulis (O. Berg) Kausel \& D. Legrand & pêra-do-mato & B & $\mathrm{N}$ & & $P$ & & & & & & & & & $\bullet$ & ○ & 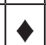 & & \\
\hline 8167 & Myrcia laruotteana Cambess ${ }^{6,9}$ & guamirim & B & $\mathrm{N}$ & & . & $\mathrm{x}$ & & & & & & & & & $\checkmark$ & & & \\
\hline 7273 & Psidium guajava $\mathrm{L}^{1,3,4}$ & goiabera & $\mathrm{S}$ & $\mathrm{N}$ & & $P$ & + & & & & & $\bullet$ & & & & & $\diamond$ & $\bullet$ & \\
\hline 8166 & Indeterminada sp.1 & & & & & . & & & & & & & & & & $\diamond$ & & & \\
\hline 8215 & Indeterminada sp.2 & & & & & . & & & & & & & & & & & $\diamond$ & & \\
\hline \multicolumn{20}{|c|}{ Onagraceae } \\
\hline 7823 & Ludwigia octovalvis (Jacq.) Raven & Cruz-de-malta & SB & $\mathrm{N}$ & & $P$ & & & & $\diamond$ & $\bullet$ & & & & & & & $\bullet$ & 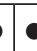 \\
\hline \multirow[t]{2}{*}{7855} & Ludwigia sp. & Cruz-de-malta & SB & $\mathrm{N}$ & & $P$ & & & & & $\checkmark$ & & & & & & & & \\
\hline & Orchidaceae & & & & & & & & & & & & & & & & & & \\
\hline 8114 & Dendrobium nobile Lindl. & orquidea & EPI & E & & $P$ & & & & & & & & & $\diamond$ & $\diamond$ & & & \\
\hline \multicolumn{20}{|c|}{ Oxalidaceae } \\
\hline 7915 & Averrhoa carambola $\mathrm{L}$. & carambola & $\mathrm{T}$ & $\mathrm{E}$ & & $P$ & & & & & $\diamond$ & & $\checkmark$ & $\diamond$ & $\diamond$ & $\diamond$ & & & - \\
\hline 8100 & Oxalis sp $1 .^{4}$ & trevo & $\mathrm{H}$ & $\mathrm{N}$ & & $\mathrm{P}$ & $\mathrm{x}$ & & & & & & & & $\diamond$ & & & & \\
\hline 8103 & Oxalis sp 2. & trevo & $\mathrm{H}$ & & & $P$ & & & & & & & & & $\diamond$ & & & & \\
\hline \multicolumn{20}{|c|}{ Passifloraceae } \\
\hline \multirow[t]{2}{*}{7913} & Passiflora amethystina Mikan & Maracujá-de-raposa & $\mathrm{CT}$ & $\mathrm{N}$ & & $P$ & & & & & $\diamond$ & $\bullet$ & & & & & & & \\
\hline & Pedaliaceae & & & & & & & & & & & & & & & & & & \\
\hline 7917 & Sesamum indicum $\mathrm{L}^{4}$ & gergelim & $\mathrm{H}$ & $\mathrm{E}$ & & $P$ & + & & & $\bullet$ & & & & $\diamond$ & & & & & \\
\hline \multicolumn{20}{|c|}{ Phytolaccaceae } \\
\hline 7532 & Seguieria guaranitica Spegazzini & & ST & $\mathrm{N}$ & & $P$ & & $\diamond$ & & & $\bullet$ & & & & & & & & \\
\hline & Piperaceae & & & & & & & & & & & & & & & & & & \\
\hline 7246 & Piper amalago $\mathrm{L}^{8}$ & parapiroba & B & $\mathrm{N}$ & & $P$ & $\mathrm{x}$ & $\bullet$ & $\bullet$ & $\bullet$ & & $\bullet$ & $\bullet$ & & & & $\diamond$ & & \\
\hline 7255 & Piper gaudichaudianum Kunth. ${ }^{8}$ & parapiroba & $\mathrm{B}$ & $\mathrm{N}$ & & $\mathrm{P}$ & $\mathrm{x}$ & & & & & & & & & & $\checkmark$ & & \\
\hline & Polygonaceae & & & & & & & & & & & & & & & & & & \\
\hline 7824 & Polygonum punctatum Elliot. & & $\mathrm{H}$ & $\mathrm{N}$ & & $P$ & & & & $\diamond$ & & & & & & & & & \\
\hline 7293 & Ruprechtia laxiflora Meissn. & & B & $\mathrm{N}$ & & . & & & & & & & & & & & $\diamond$ & & \\
\hline & Polypodiaceae & & & & & & & & & & & & & & & & & & \\
\hline 8107 & Pleopeltis angusta Humb. \& Bonpl. Ex Willd. & & $\mathrm{H}$ & $\mathrm{N}$ & & $S$ & & & & & & & & & 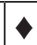 & & & & \\
\hline
\end{tabular}


TABLE I (CONTINUATION)

\begin{tabular}{|c|c|c|c|c|c|c|c|c|c|c|c|c|c|c|c|c|c|c|}
\hline \multirow{2}{*}{ HCF } & \multirow{2}{*}{ Family/Specie } & \multirow{2}{*}{ Ordinary name } & \multirow{2}{*}{$\mathbf{H b}$} & \multirow{2}{*}{$\mathbf{O}$} & \multirow{2}{*}{ SE } & \multirow{2}{*}{ B } & \multicolumn{12}{|c|}{ Months } \\
\hline & & & & & & & $\mathbf{J}$ & $\mathbf{F}$ & $\mathbf{M}$ & $\mathbf{A}$ & $\mathbf{M}$ & $\mathbf{J}$ & $\mathbf{J}$ & $\mathbf{A}$ & $\mathbf{S}$ & $\mathbf{O}$ & $\mathbf{N}$ & $\mathbf{D}$ \\
\hline & Portulacaceae & & & & & & & & & & & & & & & & & \\
\hline 7398 & Talinum paniculatum (Jacq.) Gaertn. & & $\mathrm{H}$ & $\mathrm{E}$ & $\mathrm{S}$ & & & $\bullet$ & $\diamond$ & & & & & & & & $\diamond$ & $\bullet$ \\
\hline \multicolumn{19}{|c|}{ Rhamnaceae } \\
\hline 7825 & Gouania ulmifolia Hook. et Arn. & & $\mathrm{CT}$ & $\mathrm{N}$ & $\mathrm{P}$ & & $\bullet$ & & $\bullet$ & - & $\bullet$ & - & & & & & & \\
\hline 7272 & ${\text { Hovenia dulcis } \text { Thunberg }^{4}}^{4}$ & uva-japão & $\mathrm{T}$ & $\mathrm{E}$ & $\mathrm{P}$ & + & - & $\bullet$ & & & & & & & & $\diamond$ & $\bullet$ & \\
\hline \multicolumn{19}{|c|}{ Rosaceae } \\
\hline 7649 & Eriobotrya japonica Lindley & nespera; ameixa-amarela & $\mathrm{T}$ & $\mathrm{E}$ & $\mathrm{P}$ & & & $\diamond$ & $\diamond$ & & $\diamond$ & $\bullet$ & & & & $\bullet$ & & \\
\hline 8005 & Prunus mume Sielbold \& Zucc. & umê & $\mathrm{T}$ & $\mathrm{E}$ & $\mathrm{P}$ & $\mathrm{x}$ & & & & & & & $\diamond$ & & & & & \\
\hline 7829 & Prunus persica (L.) Batsch. ${ }^{4,5}$ & pessegueiro & $\mathrm{T}$ & $\mathrm{E}$ & $\mathrm{P}$ & + & & & $\diamond$ & $\bullet$ & $\bullet$ & & & $\bullet$ & & & & \\
\hline 7275 & Prunus sellowii Koehne & pessegueiro-bravo & $\mathrm{T}$ & $\mathrm{N}$ & $\mathrm{P}$ & + & & & 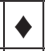 & $\bullet$ & 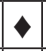 & $\bullet$ & & $\bullet$ & $\bullet$ & $\diamond$ & $\bullet$ & \\
\hline 8218 & Rubus ulmifolius Schott & groselha & B & $\mathrm{N}$ & $P$ & & & & & & & $\diamond$ & & & & $\diamond$ & $\bullet$ & \\
\hline 7295 & Rubus urticaefolius Poiret & amora-silvestre & $\mathrm{B}$ & $\mathrm{N}$ & $\mathrm{P}$ & & $\diamond$ & $\downarrow$ & $\downarrow$ & $\bullet$ & & & & & $\diamond$ & $\diamond$ & & \\
\hline \multicolumn{19}{|c|}{ Rubiaceae } \\
\hline 7396 & Manettia luteo-rubra (Vell.) Benth. & & & $\mathrm{N}$ & $\mathrm{P}$ & & $\bullet$ & $\bullet$ & & $\bullet$ & - & & & - & & & $\diamond$ & \\
\hline 7258 & Psychotria carthagenensis Jacq. ${ }^{3}$ & café-de-bugre & $\mathrm{AB}$ & $\mathrm{N}$ & $\mathrm{Si}$ & + & & 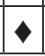 & 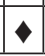 & $\bullet$ & $\bullet$ & $\bullet$ & $\bullet$ & $\bullet$ & $\bullet$ & $\bullet$ & $\bullet$ & \\
\hline \multicolumn{19}{|c|}{ Rutaceae } \\
\hline 8001 & Citrus limonia Osbeck ${ }^{3,4,5,9}$ & limão-rosa & S & $\mathrm{E}$ & $\mathrm{P}$ & + & & & $\diamond$ & $\diamond$ & & 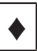 & $\diamond$ & & $\diamond$ & & & \\
\hline 8006 & Citus sinensis (L.) Osbeck ${ }^{1,4,6,9}$ & laranja & $\mathrm{T}$ & $\mathrm{E}$ & $\mathrm{P}$ & + & & & & & & & $\diamond$ & 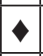 & & & & \\
\hline 8002 & Pilocarpus pennatifolius Lemaire & & $\mathrm{S}$ & $\mathrm{N}$ & $\mathrm{S}$ & & & & $\bullet$ & $\bullet$ & & $\checkmark$ & 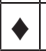 & $\diamond$ & $\bullet$ & $\bullet$ & & \\
\hline 8165 & Zanthoxylum caribaeum Lam. ${ }^{14,8}$ & mamica-de-porca & $\mathrm{T}$ & $\mathrm{N}$ & $\mathrm{P}$ & $\mathrm{x}$ & & & & & & $\diamond$ & & & & & & \\
\hline 8174 & Zanthoxylum fagara (L.) Sarg. ${ }^{4,8}$ & Mamica-de-porca & $\mathrm{T}$ & $\mathrm{N}$ & $\mathrm{P}$ & $\mathrm{x}$ & & & & & & & & & $\bullet$ & & & \\
\hline \multicolumn{19}{|c|}{ Sapindaceae } \\
\hline 8102 & Allophylus edulis (St.Hil.)Radlk. ${ }^{4,8}$ & vacum, chau-chau & $\mathrm{S}$ & $\mathrm{N}$ & $\mathrm{S}$ & + & & & $\bullet$ & & & & & 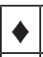 & 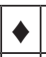 & $\bullet$ & & \\
\hline 7544 & Cardiospermum grandiflorum Sweet. & saco-de-padre & $\mathrm{CT}$ & $\mathrm{N}$ & $\mathrm{P}$ & & & & & & & & $\checkmark$ & $\bullet$ & $\diamond$ & & & \\
\hline 7923 & Cupania vernalis Camb. & camboatá & $\mathrm{T}$ & $\mathrm{N}$ & $\mathrm{Si}$ & & & & $\diamond$ & $\diamond$ & $\diamond$ & & $\bullet$ & $\bullet$ & $\bullet$ & - & & \\
\hline 8212 & Matayba elaeagnoides Radlk. & camboatá-branco & $\mathrm{T}$ & $\mathrm{N}$ & $\mathrm{Si}$ & + & & & & $\bullet$ & $\bullet$ & & $\bullet$ & & $\bullet$ & 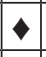 & $\bullet$ & $\bullet$ \\
\hline 7271 & Paullinia meliifolia Adr.juss. & & & $\mathrm{N}$ & $\mathrm{P}$ & & & & & & & & & & & 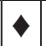 & & \\
\hline 7854 & Serjania acoma Radlk & cipó-timbó & $\mathrm{CT}$ & $\mathrm{N}$ & $\mathrm{P}$ & & & & & $\downarrow$ & & & & & & & & \\
\hline 7922 & Serjania multiflora Camb. ${ }^{6,9}$ & cipó-timbó & $\mathrm{CT}$ & $\mathrm{N}$ & $\mathrm{Si}$ & $\mathrm{x}$ & & & & $\diamond$ & $\checkmark$ & & $\bullet$ & $\bullet$ & $\bullet$ & & & \\
\hline \multicolumn{19}{|c|}{ Sapindaceae } \\
\hline 7524 & Serjania reticulata Cambess. & cipó-timbó & $\mathrm{CT}$ & $\mathrm{N}$ & $\mathrm{Sl}$ & & & & & & & & & & & $\diamond$ & & \\
\hline \multicolumn{19}{|c|}{ Scrophulariaceae } \\
\hline 7981 & Paulownia tomentosa (Thunb.)Steud. & kiri & $\mathrm{T}$ & $\mathrm{E}$ & $\mathrm{P}$ & & & & & $\bullet$ & $\diamond$ & 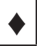 & $\downarrow$ & & $\bullet$ & & & \\
\hline \multicolumn{19}{|c|}{ Simaroubaceae } \\
\hline 7991 & Castela tweedii Planchon & & $\mathrm{B}$ & $\mathrm{N}$ & $\mathrm{P}$ & & & & & $\bullet$ & & $\diamond$ & $\bullet$ & $\bullet$ & & & & \\
\hline \multicolumn{19}{|c|}{ Solanaceae } \\
\hline 7537 & $\begin{array}{l}\text { Brugmansia suaveolens (Willd.) } \\
\text { Bercht. \& Presl. }\end{array}$ & copo-de-leite & B & $\mathrm{E}$ & $\mathrm{Si}$ & & $\diamond$ & & & & & & & - & $\bullet$ & & & \\
\hline 7379 & Capsicum baccatum $\mathrm{L}$. & pimenteira & B & $\mathrm{N}$ & $\mathrm{P}$ & & & & $\bullet$ & $\bullet$ & & & & & & & $\diamond$ & \\
\hline 8062 & Cestrum corymbosum Sch. & coerana & B & $\mathrm{N}$ & $\mathrm{Si}$ & & & & & & & & & $\diamond$ & & & & \\
\hline 7502 & Cestrum strigillatum Ruiz \& Pavón & coerana & SB & $\mathrm{N}$ & $\mathrm{P}$ & & $\bullet$ & & $\bullet$ & $\bullet$ & $\bullet$ & & & $\bullet$ & $\diamond$ & - & $\bullet$ & $\checkmark$ \\
\hline 7249 & Solanum americanum Mill. ${ }^{4}$ & maria-preta & $\mathrm{H}$ & $\mathrm{N}$ & $\mathrm{P}$ & + & $\diamond$ & $\bullet$ & $\bullet$ & & $\bullet$ & & $\bullet$ & - & & 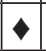 & $\bullet$ & \\
\hline 7387 & Solanum atropurpureum Schrank $^{2,3}$ & jurubeba & $\mathrm{H}$ & $\mathrm{N}$ & $\mathrm{P}$ & $\mathrm{x}$ & & & $\bullet$ & & & & & & & & $\checkmark$ & \\
\hline
\end{tabular}


TABLE I (CONTINUATION)

\begin{tabular}{|c|c|c|c|c|c|c|c|c|c|c|c|c|c|c|c|c|c|c|}
\hline \multirow{2}{*}{ HCF } & \multirow{2}{*}{ Family/Specie } & \multirow{2}{*}{ Ordinary name } & \multirow{2}{*}{$\mathbf{H b}$} & \multirow{2}{*}{$\mathbf{O}$} & \multirow{2}{*}{ SE } & \multirow{2}{*}{ B } & \multicolumn{12}{|c|}{ Months } \\
\hline & & & & & & & $\mathbf{J}$ & $\mathbf{F}$ & $\mathbf{M}$ & $\mathbf{A}$ & $\mathbf{M}$ & $\mathbf{J}$ & $\mathbf{J}$ & $\mathbf{A}$ & $\mathbf{S}$ & $\mathbf{O}$ & $\mathbf{N}$ & $\mathbf{D}$ \\
\hline 7279 & Solanum mauritianum Scop. ${ }^{2,3}$ & fumo-bravo & $\mathrm{T}$ & $\mathrm{N}$ & $\mathrm{Si}$ & $\mathrm{x}$ & 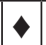 & $\bullet$ & $\diamond$ & - & & & & • & & $\diamond$ & & \\
\hline 7545 & Solanum paniculatum L., ${ }^{2,3}$ & jurubeba & B & $\mathrm{N}$ & $\mathrm{Si}$ & $\mathrm{x}$ & 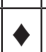 & & & & & $\bullet$ & & & & & $\bullet$ & \\
\hline 7543 & Solanum sanctae-catharinae Dunal. ${ }^{2,3}$ & juá-manso & $\mathrm{S}$ & $\mathrm{N}$ & $\mathrm{P}$ & $\mathrm{x}$ & 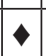 & & $\bullet$ & & & & & $\bullet$ & & $\bullet$ & & \\
\hline 7248 & Solanum sisymbriifolium Lam. & juá & SB & $\mathrm{N}$ & $P$ & $\mathrm{x}$ & & $\bullet$ & & $\bullet$ & & & & & $\diamond$ & 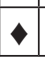 & $\bullet$ & $\bullet$ \\
\hline 7551 & Vassobia breviflora (Sandtn.) A.T. Hunziker & & & $\mathrm{N}$ & $\mathrm{P}$ & & 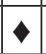 & & & & & & & & & & & \\
\hline 8063 & Indeterminada sp. ${ }^{1}$ & & & & . & & & & & & & & & $\diamond$ & & & & \\
\hline \multicolumn{19}{|c|}{ Symplocaceae } \\
\hline 8216 & Symplocos uniflora (Pohl.) Benth. & Orelha-de-onça & $\mathrm{T}$ & & . & & & & & & & & & & & $\diamond$ & & \\
\hline \multicolumn{19}{|c|}{ Tiliaceae } \\
\hline 8003 & Heliocarpus americanus L. ${ }^{5}$ & jangada & B & $\mathrm{N}$ & $\mathrm{P}$ & + & & & & & & $\bullet$ & $\diamond$ & $\bullet$ & & & & \\
\hline 7642 & Luehea divaricata Mart. ${ }^{4}$ & açoita-cavalo & $\mathrm{T}$ & $\mathrm{N}$ & $\mathrm{P}$ & + & & $\bullet$ & $\bullet$ & $\bullet$ & $\bullet$ & & & & & & & \\
\hline \multicolumn{19}{|c|}{ Ulmaceae } \\
\hline 7263 & Trema micrantha $\left(\right.$ L.) Blume ${ }^{4}$ & pau-de-polvora & $\mathrm{B}$ & $\mathrm{N}$ & $\mathrm{P}$ & + & $\bullet$ & $\bullet$ & $\diamond$ & $\bullet$ & $\bullet$ & & & $\bullet$ & $\diamond$ & $\diamond$ & $\bullet$ & $\bullet$ \\
\hline \multicolumn{19}{|c|}{ Urticaceae } \\
\hline 7259 & Boehmeria caudata $\mathrm{Sw}$. & & B & $\mathrm{N}$ & $\mathrm{P}$ & & & $\bullet$ & $\bullet$ & $\bullet$ & & & & & $\bullet$ & $\diamond$ & & \\
\hline 7501 & Urera baccifera (L.) Gaudich ex Wedd. & urtiga-mansa & $\mathrm{B}$ & $\mathrm{N}$ & $\mathrm{P}$ & & & $\bullet$ & & $\bullet$ & & & & & & & $\bullet$ & $\diamond$ \\
\hline \multicolumn{19}{|c|}{ Verbenaceae } \\
\hline 8061 & Aegiphila mediterranea Vell. & tamanqueiro & $\mathrm{T}$ & $\mathrm{N}$ & $\mathrm{P}$ & & & & & & & & & 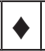 & & $\bullet$ & & \\
\hline 7389 & Aegiphila sp. & & $\mathrm{T}$ & $\mathrm{N}$ & $P$ & & & & & & & & & & & & $\diamond$ & \\
\hline 7269 & Aloysia virgata (R. \& P.) A.L. Juss. ${ }^{3,4}$ & lixeirinha & B & $\mathrm{N}$ & $\mathrm{P}$ & + & & & & $\diamond$ & & $\bullet$ & & $\diamond$ & $\diamond$ & $\diamond$ & & \\
\hline 7374 & Clerodendron x speciosum Tiejism. \& Binn. ${ }^{4}$ & lágrima-de-n. senhora & ST & $\mathrm{E}$ & $P$ & $\mathrm{x}$ & & $\diamond$ & & - & $\bullet$ & & & & & $\bullet$ & $\diamond$ & \\
\hline 7382 & Duranta repens $\mathrm{L}^{4}$ & pingo-de-ouro & $\mathrm{B}$ & $\mathrm{N}$ & $\mathrm{P} / \mathrm{Si}$ & + & $\diamond$ & $\diamond$ & $\bullet$ & $\bullet$ & $\diamond$ & & $\bullet$ & & & & $\diamond$ & \\
\hline 7256 & Lantana camara $\mathrm{L}^{4}$ & lantana & B & $\mathrm{N}$ & $\mathrm{P}$ & + & 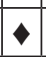 & $\bullet$ & $\checkmark$ & - & $\bullet$ & & & $\bullet$ & $\diamond$ & $\diamond$ & $\bullet$ & \\
\hline 8162 & Petrea subserrata Cham. $^{4}$ & petrea & B & $\mathrm{N}$ & Sl & $\mathrm{x}$ & & $\bullet$ & $\bullet$ & & & & & $\bullet$ & $\diamond$ & $\bullet$ & & \\
\hline 7294 & Stachytarpheta cayennensis (L.C.Rich)Vahl. & & $\mathrm{H}$ & $\mathrm{N}$ & $\mathrm{S}$ & & & & $\bullet$ & & & & & $\bullet$ & & $\diamond$ & $\bullet$ & \\
\hline \multicolumn{18}{|c|}{ Violaceae } & \\
\hline 7550 & Hybanthus bigibbosus (A. St. Hil.)Hassl. & canela-de-veado & B & $\mathrm{N}$ & $\mathrm{Si}$ & & $\diamond$ & $\bullet$ & & $\bullet$ & $\bullet$ & $\bullet$ & $\bullet$ & $\bullet$ & $\bullet$ & & $\bullet$ & \\
\hline 7546 & Hybanthus communis (A. St. Hil.)Taub. & canela-de-veado & $\mathrm{H}$ & $\mathrm{N}$ & $\mathrm{Si}$ & & $\Delta$ & 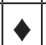 & $\diamond$ & - & $\bullet$ & & & & & $\bullet$ & $\bullet$ & \\
\hline
\end{tabular}

Plants of apiarist importance in the South and southeast regions: ${ }^{1}$ Cortopassi-Laurino and Ramalho (1988); ${ }^{2}$ Ramalho et al. (1990); ${ }^{3}$ Carvalho et al. (1999); ${ }^{4}$ Marchini et al. (2001); ${ }^{5}$ Agostini and Sazima (2003); ${ }^{6}$ Bastos et al. (2003); 7Barth (2004); ${ }^{8}$ Luz et al. (2007); ${ }^{9}$ Mendonça et al. (2008). $\mathrm{Hb}=$ Habit: $\mathrm{B}=$ bush, $\mathrm{CT}=$ climber with tendrils, $\mathrm{Ep}=$ epiphyte, $\mathrm{H}=$ herb, $\mathrm{S}=$ small tree, $\mathrm{SB}=$ sub-bush, $\mathrm{ST}=$ stem twiners, $\mathrm{T}=$ three; $\mathrm{O}=$ origin place: $\mathrm{N}$ = native species; $\mathrm{E}=$ exotic species; $\mathrm{SE}=$ Ecological succession: $\mathrm{P}=$ pioneer, $\mathrm{S}=$ secondary, $\mathrm{Si}=$ initial secondary, $\mathrm{Sl}=$ late secondary; $\mathrm{B}=$ bee plant: species $(+)$ or genera $(\times)$ reported in the literature as with apiarist importance; $\$=$ Plants photographed and collected in this work; - = Plants collected to Paraná state, with Register at the Herbarium of the Universidade Tecnológica Federal do Paraná in Campo Mourão (HCF).

\section{POLLEN ANALYSES}

Considering the three areas, a total of 30 samples of honey were analyzed and 80 pollen types distributed in 31 families were identified. Three pollen types were not identified (Table II). Microscopic images of some pollen types verified in this work are shown in Figure 2. From the taxa present in the samples, $42(52.5 \%)$ are of native species collected in the surrounding areas of the apiaries. Asteraceae and Fabaceae were the most frequent pollen types with 11 and 6 types, respectively. Euphorbiaceae and Sapindaceae presented five types each one and Mimosaceae and Myrtaceae, four types. 


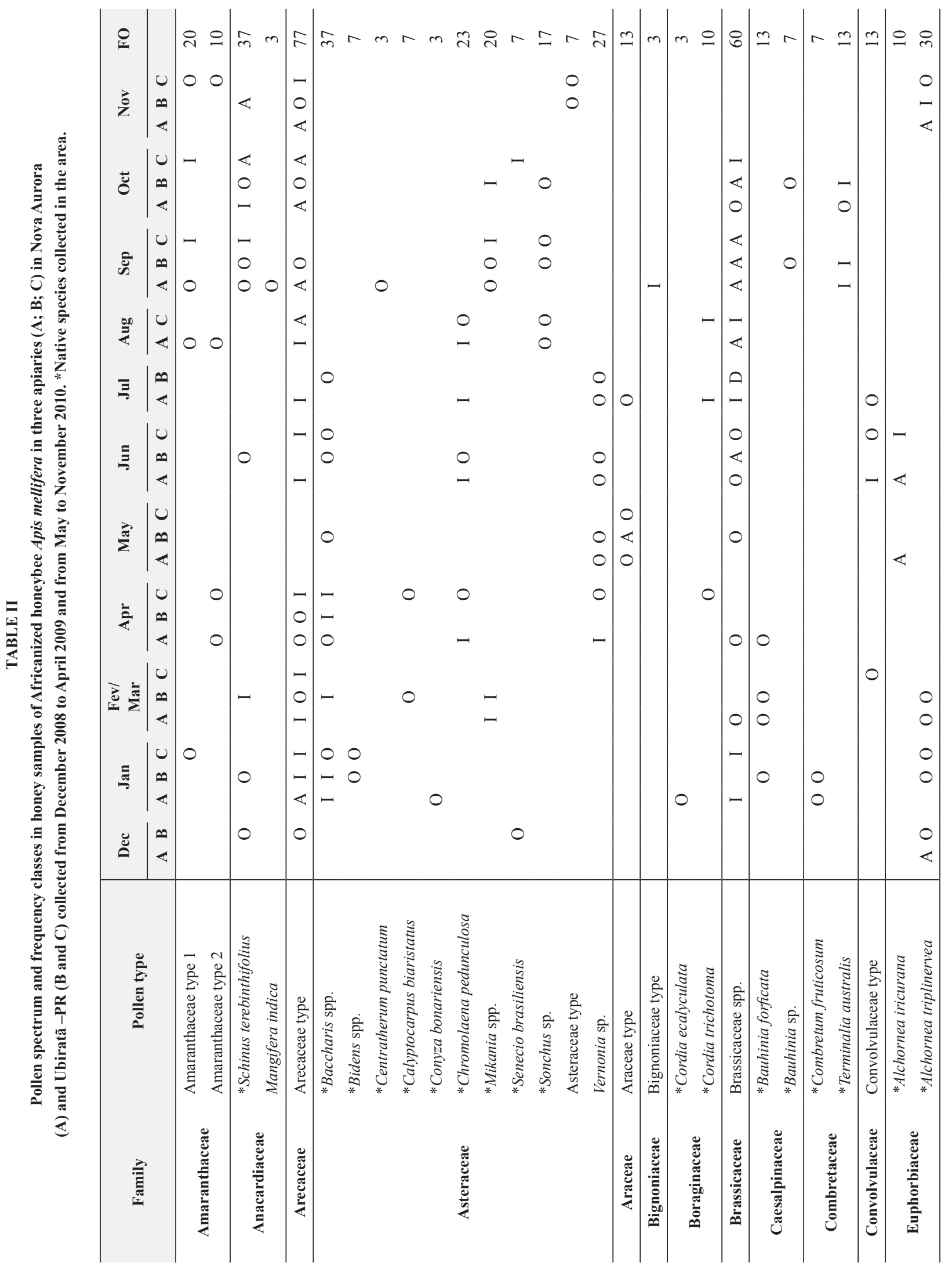




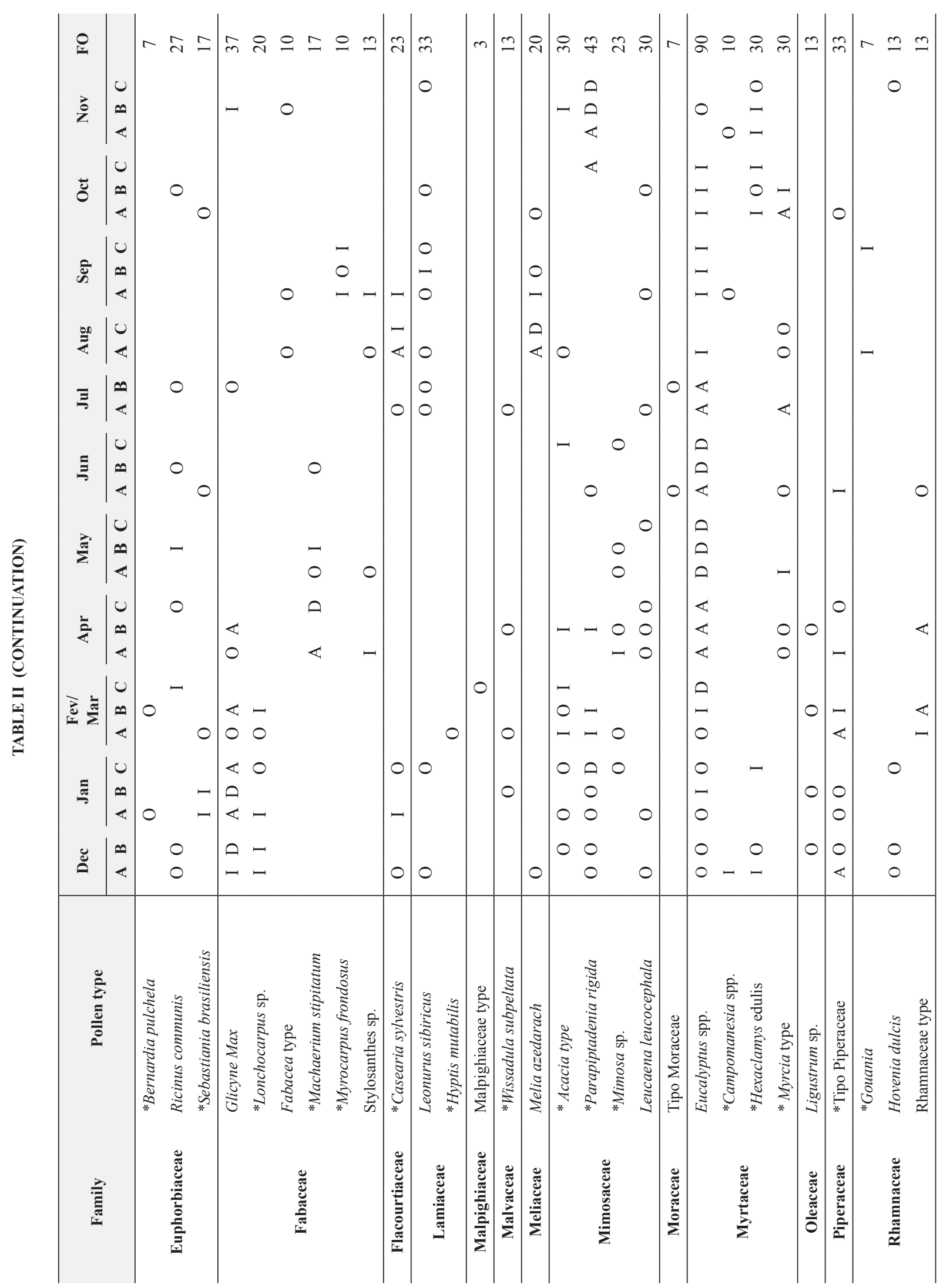


ELIZABETE S. SEKINE et al.

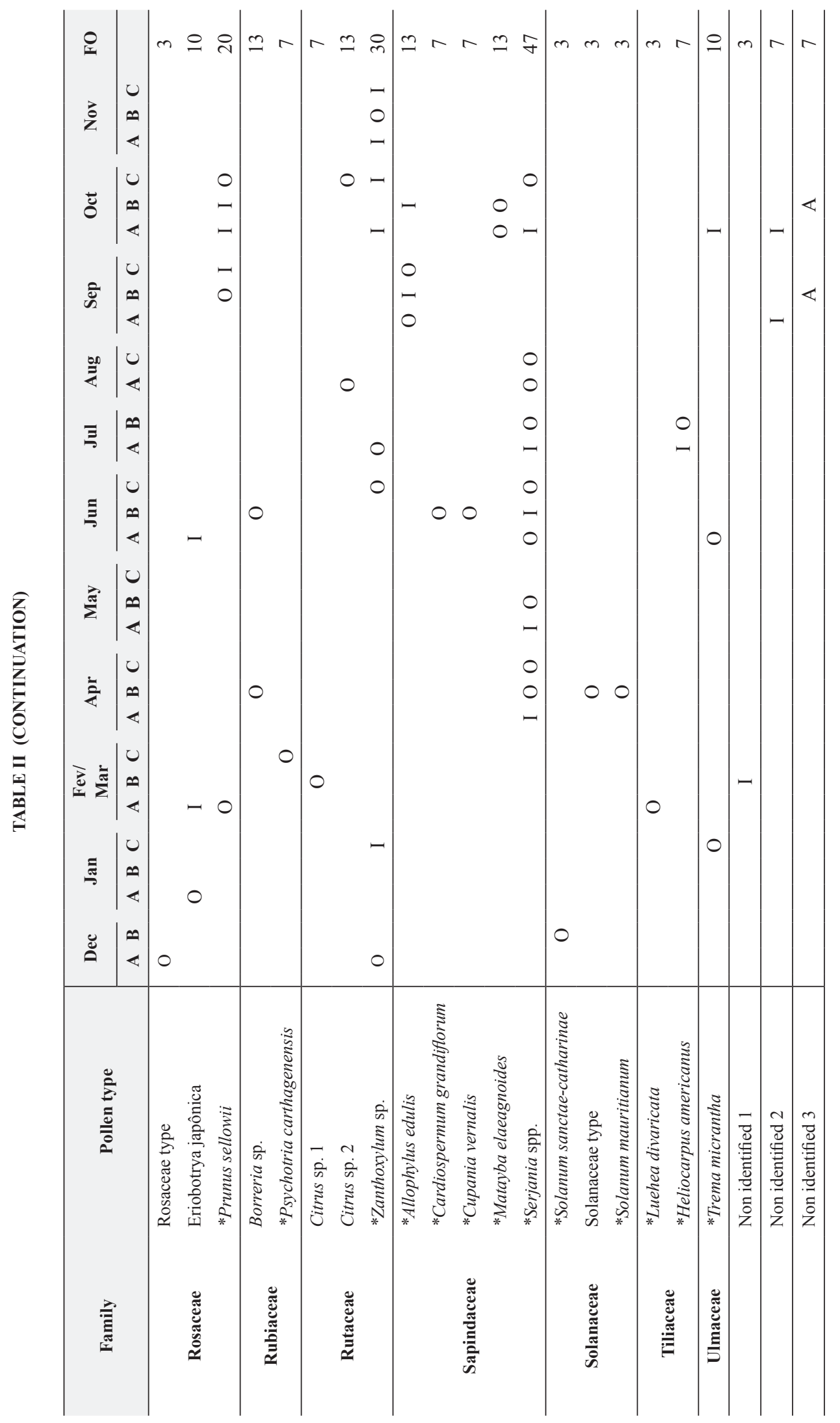


Five pollen types identified in the samples are not present in the list of the plants collected in the area: Arecaceae sp. 1, Araceae sp. 1, Stylosanthes sp., Lygustrum sp, Borreria sp.,
Acacia type and Vernonia sp. Plants of Arecaceae family and of the Herbarium.

Microscopic images recorded of same pollen types are show in Figure 2.

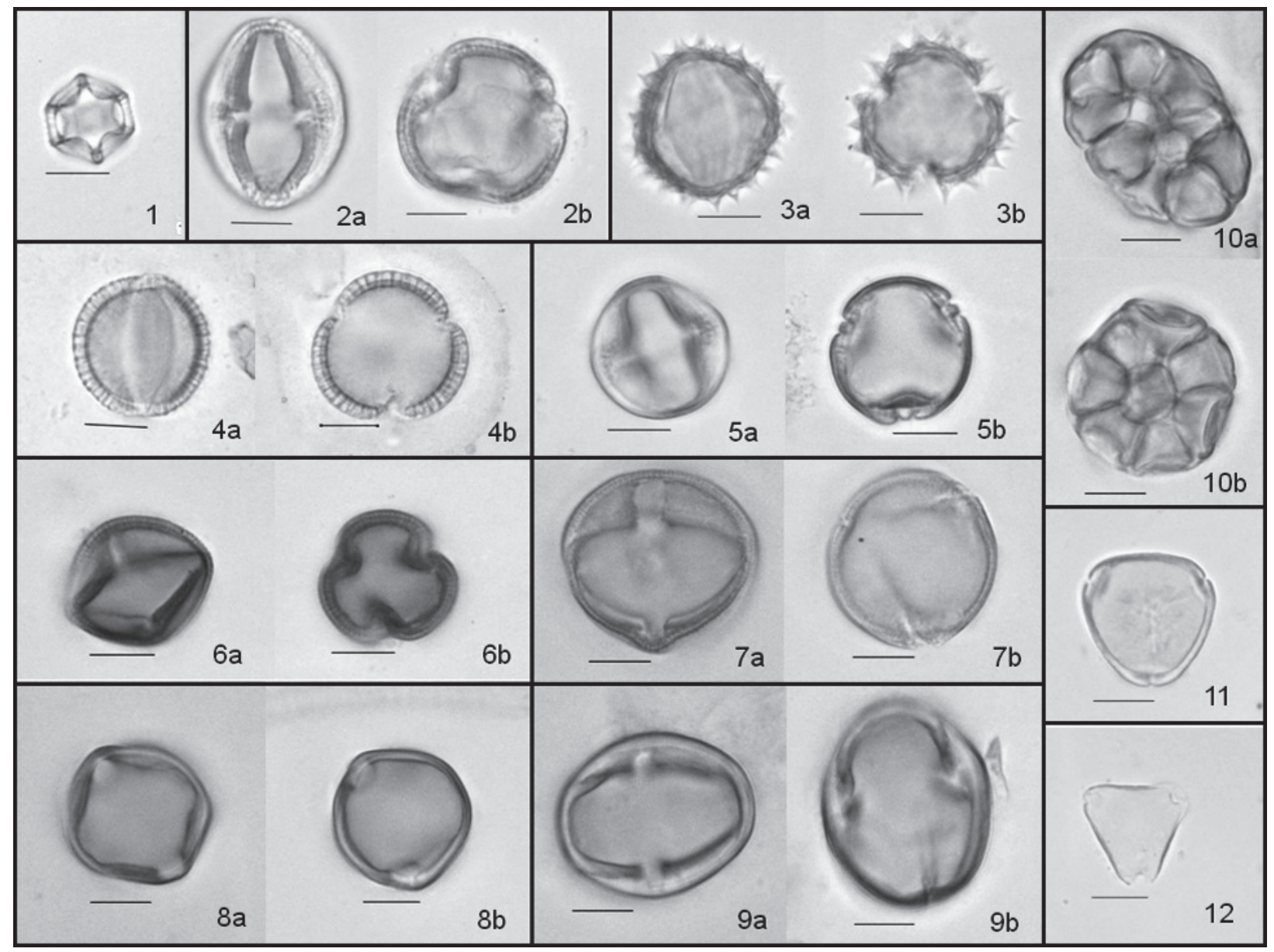

Fig 2 - Pollen types from honey samples collected in counties of Nova Aurora and Ubiratã -PR from December 2008 to May 2009. 1- Amaranthaceae sp. 1; 2- Anacardiaceae Schinus terebinthifolius; 3- Asteraceae Conyza bonariensis; 4- Brassicaceae-Raphanus sativus; 5- Euphorbiaceae Alchornea sp.; 6- Euphorbiaceae Bernardia pulchella; 7- Euphorbiaceae Ricinus communis; 8- Fabaceae Glycine max; 9- Flacourtiaceae Casearia sylvestris; 10- Mimosaceae Parapiptadenia rigida; 11- Myrtaceae Campomanesia sp.; 12- Myrtaceae Hexaclamys edulis. Scale: $10 \mu \mathrm{m}$.

Dominant pollen types were observed in 14 (47\%) of 30 honey samples, Glycine max (soybean) in December/January, Eucalyptus spp. in February/ March, May and June, Machaerium stipitatum in April, Brassicaceae sp1. in July, Melia azedarach (santa-bárbara) in August and Parapiptadenia rigida in November. Only one of the samples of the apiary A, with the largest forest area, presented the dominant pollen type of Eucalyptus in May.
The dominant pollen types G. max, Eucalyptus spp. and Brassicaceae, cultivated or ruderal plants, were found in the months with the lowest number of species collected, December, January, May, June and July (Figure 3).

The frequency of occurrence (FO) indicates that only three pollen types were present in more than $50 \%$ of the samples: Eucalyptus sp, Arecaceae sp and Brassicaceae sp. Among them, Eucalyptus 


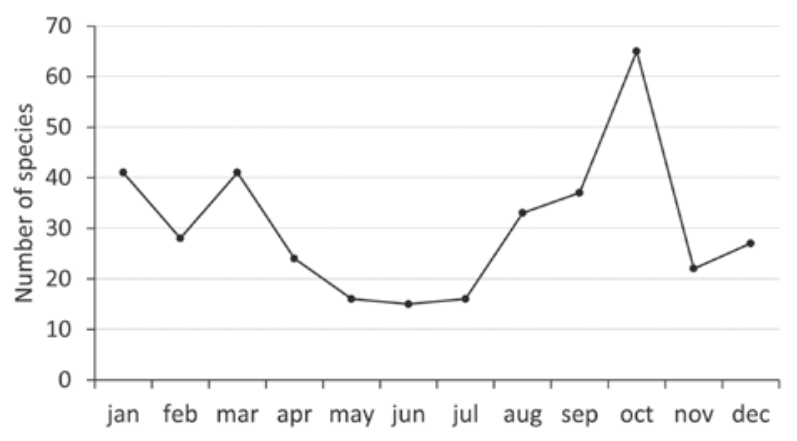

Figure 3 - Number of floristic species collected in the surrounding areas of three apiaries in the counties of Nova Aurora and Ubiratã (PR) from October 2008 to November 2009.

spp. was present as accessory or dominant pollen from February to July in 12 (40\%) of the samples.

Twelve other pollen types were also frequent, present in 30 of $50 \%$ of the samples: Schinus terebinthifolius, Baccharis spp., Alchornea triplinervea, Glycine max, Leonurus sibiricus, type Acacia, Parapiptadenia rigida, Leucaena leucocephala, Hexaclamys edulis, type Myrcia, Piperaceae sp1., Zanthoxylum sp. and Serjania spp.

Only $14(17.7 \%)$ of the total species showed frequency of more than $15 \%$ (accessory or dominant pollen) at least in one sample.

Pollen diversity calculated by the Index of Diversity of Shannon (Table III) indicates that the samples of the apiaries A and B presented higher diversity in relation to the samples of the apiary $\mathrm{C}$.

TABLE III

Index of Diversity of Shannon and Simpson based on pollen data from samples of three apiaries located in the counties of Nova Aurora (A) and Ubiratã-PR (B and C).

\begin{tabular}{l|l|l|l}
\hline Index & Apiary A & Apiary B & Apiary C \\
\hline Shannon & $\mathbf{3 . 8 9 3}$ & $3.770_{\mathrm{a}}$ & $3.603_{\mathrm{b}}$ \\
\hline Simpson & $\mathbf{0 . 9 7 5}$ & 0.971 & 0.967 \\
\hline
\end{tabular}

Values followed by the same letters, in the same line, did not differ among themselves at the level of $5 \%$ of significance.

\section{DISCUSSION}

The area of study embraces rural areas that present different types of landscape, including pastures, cultivated areas and forest remnants. The results were similar to Sakuragui et al. (2011) in a Semidecidous Tropical Forest modified by the anthropic action, in which it was observed predominance of Asteraceae, Rubiaceae, Solanaceae and Fabaceae; and Gasparino et al. (2006) that noted Asteraceae, Leguminosae and Solanaceae among the predominant families in the seeds banks of areas with riparian vegetation in a county of the west region of Paraná State.

From 34 exotic species collected in the area of study, seven were classified as exotic invader species in accordance with the administrative rule 125/2009 of the Environmental Institute of Paraná (PARANÁ 2009) that recognizes the official list of the exotic invader species to Paraná State. These species are: Leucaena leucocephala (leucena), Melia azedarach (santa-bárbara), Psidium guajava (goiaba), Ricinus communis (mamona), Tecoma stans (amarelinho), Eriobotrya japonica (nêspera) and Hovenia dulcis (uva-japão). Some of them, such as $R$. communis, M. azedarach and L. leucocephala, were representative in the pollen spectrum of the honey collected in the region.

Families with higher number of species in an area are also the main sources of nectar and pollen (Ramalho et al. 1990). Asteraceae and Labiatae species are very numerous in open vegetation areas in South America. Solanaceae, Euphorbiaceae, Palmae and Myrtaceae families are common in neotropical regions.

Asteraceae is one of the families that have the greatest number of species of apiarist importance, as this is one of the families with more species and more widely distributed among the angiosperms (Locatelli and Machado 2001).

The families of plants with better representation in honey samples analysed in this research are similar to Asteraceae, Caesalpiniaceae (Fabaceae), Malvaceae and also Myrtaceae, observed by Carvalho et al. (1999) in São Paulo State as the best representatives; and Asteraceae, Myrtaceae, Mimosaceae and Verbenaceae found by Marchini et al. (2001) as the most representative families in São Paulo State. 
Bastos et al. (2003) reported the predominance of pollen of Asteraceae, Euphorbiaceae and Leguminosae (nowadays this one is divided into Caesalpiniaceae, Fabaceae and Mimosaceae) in an area of Cerrado in Minas Gerais State. Luz et al. (2007) reported the predominance of pollen types of Arecaceae, Asteraceae, Mimosaceae and Myrtaceae in honey samples of Rio de Janeiro State. Euphorbiaceae and Myrtaceae were also found as dominant or accessory pollen types in organic honey samples collected in islands located in the frontier of Paraná, São Paulo and Mato Grosso do Sul states, and Mimosaceae, Myrtaceae and Solanaceae in nonorganic honey samples (Sereia et al. 2011).

In general, the most representative families observed in honey samples of this study are similar to those verified in samples collected in other regions of the country that have similar vegetation.

From dominant pollen types, G. $\max$ (soybean) and Eucalyptus are from plants cultivated in the area. The two counties are located in agricultural regions where the main crops are corn and soybean. In 2008, the soybean harvest in these counties was 79,950 ha (Ipardes 2010). Chiari et al. $(2005,2008)$ studied the pollination in soybean by $A$. mellifera and concluded that the visiting of flowers by these honeybees increases the production of this crop in some varieties.

Eucalyptus species are efficient sources to the formation of one type of honey approved by the customers and is the most frequent in the Brazilian market (Komatsu et al. 2002, Sodré et al. 2003). The contribution of Eucalyptus as dominant pollen in honey samples was verified in others researches developed in the southeast region by Bastos et al. (2003) in Cerrado in the State of Minas Gerais; by Barth et al. (2005) in samples of the states of São Paulo and Minas Gerais; by Luz et al. (2007) in samples of the State of Rio de Janeiro and by Mendonça et al. (2008) in samples of the State of São Paulo. Pollen grains of Eucalyptus and Baccharis are among the cited types as geographical indicators of honey of the south of Brazil (Ramalho et al. 1991).
Highly eusocial bees, such as A. mellifera, collect resources preferentially in plants that exhibit a mass-flowering syndrome, with numerous flowers opening in short time (Wilms et al. 1996). This feature can be observed in many cultivated plants and invader species, explaining the occurrence of these pollen types in various samples, even as dominant pollen in some months.

In this research, only $17.7 \%$ of the pollen types showed frequency higher than $15 \%$ at least in one honey sample. Cortopassi-Laurino and Ramalho (1988), analyzing samples of pollen collected by Apis mellifera on the outskirts of São Paulo city, also observed a low percentage of plants whose pollen accounted for more than $10 \%$ in the samples.

Sources of pollen with representativeness between $1 \%$ and $10 \%$ are resources with little attractiveness, corresponding to potential or secondary sources. These sources supplement the nutritional needs of the colony and may be important in environments where food resources suffer seasonal variations (Ramalho and Kleinert-Giovannini 1986).

The large number of pollen types with low frequency in the samples shows the importance of these plants in total honey production, representing a significant resource for the beekeeping of the region.

Bees have a highly developed sense of smell and they can be trained to look for a flavor or a particular mix of aromas. Once they become conditioned to seek a kind of plant, they will continue to get it (Free 1993). Bees exhibit floral constancy, as a tendency to use fewer species than would be expected considering the number of flowering plants at the site (Cane and Sipes 2006). However, according to Wilms et al. (1996) Africanized bees are also quite general in the exploitation of floral resources, representing a wider niche than other eusocial bees.

The high values verified by the application of the Simpson Index indicate the probability of diversity of the sampled pollen, due to the dominance of one or a few species. 
Samples of honey of this state are strongly heterofloral, and reported as frequent pollen types Allophylus, Baccharis, Campomonesia, Cecropia, Citrus, Eucalyptus, Matayba and Mimosa scabrela, Paspalum e Vernonia, with greater incidence of Eucalyptus (Ramalho et al. 1991).

Pollen grains of the type Baccharis (Asteraceae), of Euphorbiaceae and Asteraceae and type Eupatorium, were common in samples from Paraná State. Mimosa scabrela is typical from the region of Curitiba and pollen of the type Brassicaceae was also common in the samples (Carpes et al. 2009).

Approximately $52.5 \%$ of the pollen types verified in the honey samples are from native species of the region. Native species with bee value can be used in programs of recuperation of degraded areas. Although studies about this theme are not very common, Pegoraro and Ziller (2003) conducted a survey of species with bee value to provide subsidies to the restoration of a legal reserve. These authors reported some genera identified in this research, such as Mimosa, Eugenia and Zanthoxylum, as important to this purpose. Baggio (1988) and Wolff et al. (2007) indicated the application of aroeira, Schinus terebinthifolius, in agroforestry systems for beekeeping.

The use of native species by the bees was confirmed by the diversity of species found in this study, highlighting the importance of preserving native vegetation for the survival of the colonies during periods of supply scarcity of the floral resources of introduced species such as G. $\max$ and Eucalyptus spp.

\section{CONCLUSION}

In general, the honey collected in the region was heterofloral. Pollen types of cultivated crops were very representative, and the dominant pollen like Glycine max in December and January and Eucalyptus spp. in May and June. From the native species, Parapiptadenia rigida was dominant in November and February and Machaerium stipitatum in April.
Pollen types of other native species, such as Alchornea iricurana, A. triplinervea, Baccharis spp., Casearia sylvestris, Hexaclamys edulis, Schinus terebinthifolius, Serjania spp. and Zanthoxylum $\mathrm{sp}$. were found as accessory pollen. If other pollen types, found as isolated pollen, were considered in group, they were very representative in the samples, highlighting the importance of the native vegetation for the survival of the colonies.

\section{RESUMO}

O objetivo deste trabalho foi realizar um levantamento da flora com potencial apícola nos municípios de Ubiratã e Nova Aurora-PR, por meio da coleta de plantas e análises polínicas em amostras de mel coletadas mensalmente. Foram registradas 208 espécies de plantas, distribuídas em 66 famílias. As famílias que apresentaram maior riqueza de tipos polínicos foram Asteraceae, Myrtaceae e Solanaceae. Aproximadamente 80 tipos polínicos foram encontrados nas amostras de mel e, na maioria, foram caracterizados como heteroflorais. Plantas cultivadas, como Glycine max (soja) e Eucalyptus spp. foram representativas em alguns meses do ano. Espécies exóticas, tais como Ricinus communis and Melia azedarach também foram frequentes. No entanto, mais de $50 \%$ dos tipos polínicos pertencem a espécies nativas da região, tais como Schinus terebinthifolius, Baccharis spp., Alchornea triplinervea, Parapiptadenia rigida, Hexaclamys edulis, Zanthoxylum sp. and Serjania spp., indicando a importância da vegetação nativa para a sobrevivência das colônias.

Palavras-chave: apicultura, plantas apícolas, recursos florais, levantamento florístico.

\section{REFERENCES}

Agostini K And Sazima M. 2003. Plantas ornamentais e seus recursos para abelhas no campus da Universidade Estadual de Campinas, estado de São Paulo, Brasil. Bragantia 62: 335-343.

BAGGIO AM. 1988. Aroeira como potencial para usos múltiplos na propriedade rural. Bol Pesq F1 17: 25-32.

BARTH OM. 1989. O pólen do mel brasileiro. Rio de Janeiro: Luxor, $226 \mathrm{p}$.

BARTH OM. 2004. Melissopalynology in Brazil: a review of pollen analysis of honey, propolis and pollen loads of bees. Sci Agric 61: 342-350. 
BARTH OM, MAIORINO C, BENATTI APT AND BASTOS DHM. 2005. Determinação de parâmetros físico-químicos e da origem botânica de méis indicados monoflorais do sudeste do Brasil. Cienc Tecnol Aliment 25: 229-233.

Bastos EMAF, Silveira VM And SoAres AEE. 2003. Pollen spectrum of honey produced in cerrado areas of Minas Gerais state (Brazil). Braz J Biol 63: 599-615.

CANE JH AND SIPES S. 2006. Characterizing floral specialization by bees: analytical methods and a revised lexicon for oligolecty. In: WASER NMAND OLLERTON J (Eds), Plantpollinator interactions: from specialization to generalization, Chicago: University of Chicago Press, p. 99-122.

CARPES ST, CABRAL ISR, LUZ CFP, CAPELETTI JP, ALENCAR SM AND MASSON ML. 2009. Palynological and physicochemical characterization of Apis mellifera L. bee pollen in the Southern region of Brazil. J Food Agric Environ 7: 667-673.

CARvalho CAL AND MarChini LC. 1999. Plantas visitadas por Apis mellifera L. no vale do rio Paraguaçu, Município de Castro Alves, Bahia. Rev Bras Bot 22: 333-338.

CARvalho CAL, Marchini LC AND Ros PB. 1999. Fontes de pólen utilizadas por Apis mellifera L. e algumas espécies de Trigonini (Apidae) em Piracicaba (SP). Bragantia 58: 49-56.

Chiari WC, TOledo VAA, HofFmann-CAMPo CB, RuvoloTAKASUSUKI MCC, TOLEDO TCSOA AND LOPES TS. 2008. Polinização por Apis mellifera em soja transgênica [Glycine $\max ($ L.) Merrill] Roundup ReadyTM cv. BRS 245 RR e convencional cv. BRS 133 Acta Sc Agron 30: 267-271.

Chiari WC, TOledo VAAT, RuVOlO-TAKasusuki MCC, AtTencia VM, Costa FM, Kotaka CS, SAKaguti ES AND Magalhães HR. 2005. Floral Biology and Behavior of Africanized Honeybees Apis mellifera in Soybean (Glycine max L. Merril). Braz Arch Biol Technol 48: 367-378.

CORTOPASSI-LAURINO M AND RAMALHO M. 1988. Pollen harvest by Africanized Apis mellifera and Trigona spinipes in São Paulo. Botanical and ecological views. Apidologie 19: 1-24.

CRANE E. 1990. Bees and beekeeping: science, practice and world resources. Oxford: Heinemann Newness, 614 p.

CRONQUist A. 1981. An integrated system of classification of flowering plants. New York: Columbia University Press, $1262 \mathrm{p}$.

EMBRAPA - EMPRESA BRASILEIRA DE PESQUISA AGROPECUARIA. 1999. Centro nacional de pesquisa de solos. Sistema brasileiro de classificação de solos. Brasília: Embrapa, 412 p.

ERDTMAN G. 1952. Pollen morphology and plant taxonomyAngiosperms. Stockholm: Almqvist e Wiksel, 539 p.

FidAlgo O AND BonONI VLR. 1989. Técnicas de coleta, preservação e herborização de material botânico. São Paulo: Instituto de Botânica.

FREE JB. 1993. Insect pollination of crops. $2^{\text {nd }}$ ed., London: Academic Press, 684 p.

Gasparino D, Malavasi UC, Malavasi MM AND Souza I. 2006. Quantificação do banco de sementes sob diferentes usos do solo em área de domínio ciliar. Rev Árvore 30: 1-9.
HiLl D AND WeBSTER C. 1995. Apiculture and forestry (Bees and trees). Agroforestry Systems 29: 313-320.

IAPAR - InSTITUTO AgRONÔMICO DO PARANÁ. 2009. Cartas climáticas do Paraná. Acessado em: 16 nov. 2009. Disponível em: http://www.iapar.br/modules/conteudo/ conteudo.php?conteudo $=677$.

IBGE - FundaÇÃo InSTITUTO BRASILEIRO DE GEOGRAFIA E ESTATÍSTICA. 1992. Manual técnico da vegetação brasileira. Departamento de Recursos Naturais e Estudos Ambientais. - Rio de Janeiro: IBGE, 92 p.

IPARDES - INSTITUto PARANAENSE DE DESENVOLVIMENTO ECONÔMICO E SOCIAL. 2010. Cadernos Municipais. Acessado em: 16 nov. 2010. Disponível em: http:// www.ipardes.gov.br/modules/conteudo/conteudo. php? conteudo $=5$

Komatsu SS, MARChINI LC AND MoReti AC. 2002. Análises Análises físico-químicas de amostras de méis de flores silvestres, de eucalipto e de laranjeira, produzidos por Apis mellifera L., 1758 (Hymenoptera, Apidae) no estado de São Paulo: conteúdo de açúcares e de proteína. Cienc Tecnol Aliment 22: 143-146.

LOCATELLI E AND MACHADO IC. 2001. Bee diversity and their floral resources in a fragment of a tropical altitudinal wet forest ("Brejos de altitude") in Northeastern Brazil. Acta Hort 561: 317-325.

Louveaux J, Maurizio A And Vorwohl G. 1978. Methods of melissopalynology. Bee World 59: 139-157.

LUZ CFP, THOMÉ ML AND BARTH OM. 2007. Recursos tróficos de Apis mellifera L. (Hymenoptera, Apidae) na região de Morro Azul do Tinguá, Estado do Rio de Janeiro. Rev Bras Bot 30: 29-36.

Marchini LC, Moreti AC, Teixeira EW, Silva ECA, RODRIGUES RR AND SOUZA VC. 2001. Plantas visitadas por abelhas africanizadas em duas localidades do estado de São Paulo. Sci Agric 58: 413-420.

Melhem TS, Cruz-Barros MAV, CorrêA AMS, MakinoWatanabe H, Silvestre-CAPELATO MSF AND GolÇAlVEs-Esteves VL. 2003. Variabilidade Polínica em Plantas de Campos do Jordão (São Paulo, Brasil). Bol Inst Bot São Paulo 16: 1-104.

Mendonça K, Marchini LC, Souza BA, Almeida-AnaCleto D AND MORETI AC. 2008. Plantas apícolas de importância para Apis mellifera L. ( Hymenoptera: Apidae) em fragmento de Cerrado em Itirapina, SP. Neotropic Entomol 37: 513-521.

Moreti AC, CARVALHo CAL, Marchini LC AND OliveIRA PCF. 2000. Espectro polínico de amostras de mel de Apis mellifera L., coletadas na Bahia. Bragantia 59: 1-6.

Moreti AC, MARCHINI LC, SOUZA VC AND RodRIGUES RR. 2002. Atlas do pólen de plantas apícolas. Rio de Janeiro: Papel Virtual Editora, 93 p.

Nogueira Couto RH AND CoUto LA. 2006. Apicultura: manejo e produtos. $3^{\mathrm{a}}$ ed., Jaboticabal: FUNEP, $193 \mathrm{p}$.

PARANÁ. InSTITUTO AMBIENTAL DO PARANÁ - IAP. 2009. Lista de espécies exóticas invasoras do Paraná, Portaria 125/2009. Curitiba: IAP. 
PEgORARO A AND ZILLER SR. 2003. Valor apícola das espécies vegetais de duas fases sucessionais da Floresta Ombrófila Mista, em União da Vitória, Paraná-Brasil. Bol Pesq F1 47: 69-82.

PIELOU EC. 1975. Ecological diversity. New York: J Wiley, $165 \mathrm{p}$.

PoOLE RW. 1974. An introduction to quantitative ecology. New York: McGraw-Hill, 532 p.

RAMALHO M, GUIBU LS, GIANNINI TC, KLEINERT-GIOVANNINI A AND IMPERARTRIZ-FONSECA VL. 1991. Characterization of some southern Brazilian honey and bee plants through pollen analysis. J Api Res 30: 81-86.

Ramalho M And Kleinert-Giovannini A. 1986. Some aspects of the utilization of pollen analysis in ecological research. Apidologie 17: 159-174.

RAMALHO M, KLEINERT-GIOVANNINI A AND IMPERATRIZFONSECA VL. 1990. Important bee plants for stingless bees (Melipona and Trigonini) and Africanized honeybees (Apis mellifera) in neotropical habitats: a review. Apidologie 21: 469-488.

Reitz R, Klein RM AND ReIS A. 1978. Projeto madeira de Santa Catarina. Itajaí - Santa Catarina, n. 28-30, 320 p.

ReItZ R, KLEIn RM AND ReIS A. 1983. Projeto madeira do Rio Grande do Sul. Sellowia n. 34-35, 525 p.

RoderJan CV, KuniYoshi YS AND GALVÃo F. 1998. As regiões fitogeográficas do estado do Paraná. $2^{\mathrm{a}}$ ed., Curitiba: Acta For Bras v. 1.

RoubIK DW. 2010. Pollen and spores of Barro Colorado Island. Acessado em: 12 nov. Disponível em: http:// striweb.si.edu/roubik/.

SAKURAGUI CM, ALVES EM, LORENZETTI ER, JANUNZZI AM, BORGES RAX, TOLEDO VAA. 2011. Bee flora of an insular ecosystem in southern Brazil. J Bot Res Inst Texas 5: 311-319.

SANTOS RF, KIILL LHP AND ARAÚJO JLP. 2006. Levantamento da flora melífera de interesse apícola no município de Petrolina-PE. Caatinga 19: 221-227.

Sereia JA, Alves EM, Toledo VAA, Marchini LC, SeKine ES, Faquinello P, Almeida D AND Moreti AC. 2011. Physicochemical characteristics and pollen spectra of organic and non-organic honey samples of Apis mellifera L. An Acad Bras Cienc 83: 1077-1090.
SILVA SJR AND ABSY ML. 2000. Análise do pólen encontrado em amostras de mel de Apis mellifera L. (Hymenoptera, Apidae) em uma área de savana de Roraima, Brasil. Acta Amaz 30: 579-588.

Sodré GS, MARCHINI LC, MoReti AC AND CARVALHO CAL. 2003. Análises multivariadas com base nas características físico-químicas de amostras de méis de Apis mellifera L. (Hymenoptera: Apidae) da região litoral norte no estado da Bahia. Arch Latinoam Prod Anim 11: 129-137.

SODRÉ GS, MARCHINI LC, MORETI AC AND CARVALHO CAL. 2008. Tipos polínicos encontrados em amostras de méis de Apis mellifera em Picos, Estado do Piauí. Ciênc Rural 38: 839-842.

SPVS - Sociedade de Pesquisa em Vida Selvagem E EDUCAÇÃO AMBIENTAL. 1996. Nossas árvores: Manual para recuperação da reserva florestal legal. Curitiba: FNMA, 84 p.

VIANA BF, Silva FO AND KlEINERT AMP. 2006. A flora apícola de uma área restrita de dunas litorâneas, Abaeté, Salvador, Bahia. Rev Bras Bot 29: 13-25.

VieIRA GHC, MARChini LC, Souza BA AND Moreti AC. 2008. Fontes florais usadas por abelhas (Hymenoptera, Apoidea) em área de Cerrado no município de Cassilândia, Mato Grosso do Sul, Brasil. Ciênc Agrotec 32: 1454-1460.

WILMS W, IMPERATRIZ-FONSECA VL AND ENGELS W. 1996. Resource partitioning between highly eusocial bees and possible impact for the introduced Africanized honey bee on native stingless bees in the Brazilian Atlantic Rainforest. Stud Neotrop Fauna \& Environm 31: 137-151.

WolfF L, CARdoso J, SchWENGBer J AND SCHIEDECK G 2007. Sistema agroflorestal apícola envolvendo abelhas melíferas, abelhas indígenas sem ferrão, aroeira-vermelha e videiras em produção integrada no interior de PelotasRS: um estudo de caso. Rev Bras Agroecol 2: 1236-1239. 